\title{
Dynamic Consumption and Portfolio Choice under Prospect Theory*
}

\author{
Servaas van Bilsen \\ Dept. of Quantitative Economics \\ University of Amsterdam \\ and NETSPAR
}

\author{
Roger J. A. Laeven ${ }^{\dagger}$ \\ Dept. of Quantitative Economics \\ University of Amsterdam \\ EURANDOM and CentER
}

Tuesday $4^{\text {th }}$ February, 2020

\begin{abstract}
This paper explicitly derives the optimal dynamic consumption and portfolio choice of an individual with prospect theory preferences. The individual is loss averse, endogenously updates his reference level over time, and distorts probabilities. We show that the optimal consumption strategy is rather insensitive to economic shocks. In particular, in case the individual sufficiently overweights unlikely unfavorable events, our model generates an endogenous floor on consumption. As a result, an individual with prospect theory preferences typically implements a (very) conservative portfolio strategy. We discuss implications of our results for the design of investment-linked annuity products.
\end{abstract}

JEL classification: D81, D91, G02, G11.

Keywords: Loss Aversion, Endogenous Reference Level, Optimal Consumption Choice, Optimal Portfolio Choice, Probability Weighting, Optimal Annuity Design.

${ }^{*}$ We are very grateful to the editor and the referee for comments and suggestions that have significantly improved the paper. We are also grateful to Yacine Aït-Sahalia, Lans Bovenberg, Nicole Branger, Conrad Ciccotello, Louis Eeckhoudt, Martin Grace, Michael Hoy, Gur Huberman, Frank de Jong, Olivia S. Mitchell, Theo Nijman, Andreas Richter, Nikolai Roussanov, Hans Schumacher, Kent Smetters, Mogens Steffensen, Sharon Tennyson, Jeremy Tobacman, Michel Vellekoop, Wei Xiong, and to conference and seminar participants at the Australasian Finance and Banking Conference, the Foundations of Utility and Risk Conference, the S.S. Huebner Foundation Colloquium, the Netspar International Pension Workshop, the Quantitative Methods in Finance Conference, the International Congress on Insurance: Mathematics and Economics, the Center for Financial Studies at Goethe University Frankfurt, Tilburg University, the Tinbergen Institute, the University of Amsterdam, the University of Münster, and the University of Pennsylvania (Wharton School) for their helpful comments and suggestions. This research was supported in part by the Netherlands Organization for Scientific Research under grant NWO VIDI (van Bilsen, Laeven), by the TIAA-CREF Institute under grant ID\#00464942 (van Bilsen) and by the European Commission under grant EU-MOPACT (van Bilsen). Conflicts of interest: none. Email addresses: S.vanBilsen@uva.nl, and R.J.A.Laeven@uva.nl.

${ }^{\dagger}$ Corresponding author. Mailing Address: PO Box 15867, 1001 NJ Amsterdam, The Netherlands. Phone: +31 (0) 205254219 . 


\section{Introduction}

The problem of optimal consumption and portfolio choice over the life cycle has become a canonical problem in the economics and mathematics of risk, insurance and finance since Merton (1969) who was the first to analytically solve it in a continuous-time setting 11 His seminal work has been extended along many dimensions to account for, e.g., stochastic investment opportunities (see, e.g., Chacko and Viceira (2005), Liu (2007), and Laeven and Stadje (2014)), labor income (see, e.g., Bodie, Merton, and Samuelson (1992) and Benzoni, Collin-Dufresne, and Goldstein (2007)), housing costs (see, e.g., Cocco (2005)), and unexpected health expenditures (see, e.g., Edwards (2008) and Shao, Chen, and Sherris (2019)).

Many of these life-cycle papers assume standard preferences: they assume that the individual exhibits either constant relative risk aversion (CRRA) utility or Epstein-Zin utility. An extensive body of literature in behavioral economics, insurance and finance, however, documents experimental and empirical departures from the key assumptions underlying these preference models in a wide variety of risky choice situations as well as from the consumption and portfolio implications they generate. This has triggered researchers to develop a variety of alternative theories of decision-making under risk and analyze their implications for optimal consumption and portfolio choice. Some well-known alternative theories are regret theory (Loomes and Sugden (1982), Bell (1982, 1983), Sugden (1993), and Quiggin (1994)), disappointment (aversion) theory (Bell (1985), Loomes and Sugden (1986), and Gul (1991)), and habit formation Abel (1990), Constantinides (1990), and Sundaresan (1989)). Their consumption and portfolio implications have been analyzed in e.g., Muermann, Mitchell, and Volkman (2006), Ang, Bekaert, and Lui (2005), Schroder and Skiadas (2002), and Bilsen, Bovenberg, and Laeven (2019a).

Another well-known alternative theory - which has received much attention in the experimental and empirical literature - is prospect theory (PT) originally proposed by Kahneman and Tversky (1979) and Tversky and Kahneman (1992). The current paper analytically solves the consumption and portfolio choice problem of an individual with

\footnotetext{
${ }^{1}$ See also the related work of Mossin (1968), Samuelson (1969), and Merton (1971).
} 
prospect theory preferences featuring loss aversion, an endogenous reference level, and probability weighting, and analyzes how these features jointly impact the optimal consumption and portfolio strategies. We make the following modeling assumptions. First, the individual derives utility from the difference between consumption and a reference level. If consumption is larger (smaller) than the reference level, then the individual experiences a gain (loss). Second, inspired by Tversky and Kahneman (1992), instantaneous preferences are represented by the so-called two-part power utility function. This function has a kink at the reference level: losses hurt more than same-sized gains satisfy inducing loss aversion. Third, the individual's reference level depends on the individual's own past consumption choices consistent with internal habit formation (see, e.g., Constantinides (1990), Detemple and Zapatero (1992), and Detemple and Karatzas (2003)). Finally, the individual distorts objective probabilities by applying probability weighting. The probability weighting functions - one for gains and one for losses - are allowed to be inverse S-shaped.2

Our intricate solution procedure consists of four steps. We first invoke the solution method developed by Schroder and Skiadas (2002). These authors show how to convert a consumption and portfolio choice problem featuring linear internal habit formation into a consumption and portfolio choice problem without habit formation. We employ the method of Schroder and Skiadas (2002) to transform the individual's original maximization problem into a dual problem without an endogenous reference level. Then, we rewrite the individual's dual problem in terms of the quantile function of surplus (or dual) consumption 3 The objective function now reduces to an ordinary linear expectation. We refer to Jin and Zhou (2008) and He and Zhou (2011) for more details on this clever insight of quantile formulation in a terminal wealth portfolio choice problem; we suitably adapt it here to our setting with intertemporal consumption. Next, in order to handle pseudo-concavity and non-differentiability, we search for the local maxima of the objective function and we determine the global maximum by comparing, in a particular way, the local maxima; see Basak and Shapiro (2001) and Berkelaar, Kouwenberg, and Post (2004)

\footnotetext{
${ }^{2}$ An extensive body of literature finds that individuals overweight extreme events and underweight normal events (see, e.g., Wu and Gonzalez (1996), Abdellaoui (2000), and Bleichrodt and Pinto (2000)). This finding implies an inverse S-shaped probability weighting function.

${ }^{3}$ Surplus consumption is defined to be the difference between consumption and the individual's reference level.
} 
who apply a similar procedure to solve a terminal wealth portfolio choice problem without intertemporal consumption. Finally, we obtain explicit closed-form solutions to our original problem by exploiting the equivalence relationships between the dual problem and the primal (original) problem.

Our results can be summarized as follows. First, we find that the individual divides the state of the economy into four categories: very good scenarios (very low state prices), good scenarios (low state prices), bad scenarios (high state prices) and very bad scenarios (very high state prices). This classification is based upon whether consumption is above or below the reference level and upon whether the individual overweights or underweights probabilities of the respective events. Second, we find that the probability weighting functions affect the sensitivity of consumption to economic shocks. For reasonable parameter choices, optimal consumption is rather insensitive to economic shocks in a wide range of scenarios. In particular, we find that if the individual sufficiently overweights unlikely unfavorable events, our preference model generates an endogenous floor on consumption. We explicitly derive the level of this floor on consumption. Probability weighting may thus explain why some individuals purchase investment-linked annuities with a guaranteed minimum income benefit (GMIB).

The optimal portfolio profile (i.e., the share of wealth invested in the risky stock as a function of the state of the economy) displays a U-shaped pattern if probabilities are not distorted. If the individual overweights unlikely unfavorable events, then the share of assets invested in the risky stock is relatively low in most scenarios. In case he also overweights probabilities of unlikely favorable events, the optimal portfolio profile is substantially larger, especially in (very) good economic scenarios.

We also compute the welfare costs associated with incorrectly assuming CRRA utility 4 More specifically, we consider an individual with prospect theory preferences who delegates his consumption and portfolio decisions to a professional asset manager (e.g., pension fund). The asset manager makes the consumption and portfolio decisions on behalf of his clients based on CRRA utility. We assume that our individual chooses a CRRA strategy such that the difference between his optimal utility (i.e., the utility level associated with the optimal life-cycle

\footnotetext{
${ }^{4}$ We measure minimum welfare losses in terms of the relative decline in certainty equivalent consumption.
} 
policies) and his actual utility (i.e., the utility level associated with the suboptimal CRRA life-cycle policies) is as small as possible. We find that endogenous updating of the reference level typically has a relatively large impact on the minimum welfare loss, while the impact of probability weighting is less pronounced. Our computations show that the minimum welfare loss can be substantial (i.e., more than 10\%) depending on the values of the preference parameters.

The extant literature on dynamic optimal consumption and portfolio choice under prospect theory preferences is still relatively scarce $5^{5}$ Gomes (2005) explores the optimal portfolio choice of a loss-averse individual in an economy with only two states of nature. Berkelaar et al. (2004) examine the optimal portfolio choice of a loss-averse individual in a setting with terminal wealth and a continuum of states of nature. Bilsen, Laeven, and Nijman (2019b) include intertemporal consumption choice and allow the individual to endogenously update his reference level over time. The model of Bilsen et al. (2019b) does, however, not accommodate probability weighting. Jin and Zhou (2008) and He and Zhou (2011) study the optimal portfolio choice of an individual that maximizes prospect theory value of terminal wealth. These authors take probability weighting into account, but do not consider intertemporal consumption choice and an endogenous reference level. The present paper considers a preference model that allows for intertemporal consumption choice, loss aversion, an endogenous reference level, as well as probability weighting. To our best knowledge, this paper is the first to allow for all these prospect theory features together and analyze their joint impact on consumption and portfolio decisions.

The remainder of this paper is structured as follows. Section 2 describes the economy and the individual's preferences, and formulates the optimization problem. Section 3 presents our solution method. An analysis of the optimal strategies, their welfare implications, and a discussion of their implications for annuity contract design, is presented in Section 4 . Section 5 concludes the paper. Proofs are relegated to the Appendix.

\footnotetext{
${ }^{5}$ Several authors use prospect theory preferences to explain stylized facts observed in financial time series. For example, Benartzi and Thaler (1995) find that loss aversion helps to explain the equity premium puzzle.
} 


\section{Financial Market, Preferences and Optimization Problem}

\subsection{Financial Market}

Let $T>0$ be a fixed terminal time. The randomness in the financial market is represented by a filtered probability space $(\Omega, \mathcal{F}, \mathbb{F}, \mathbb{P})$. On this probability space we define a standard $N$-dimensional Brownian motion $\left\{Z_{t}\right\}_{0 \leq t \leq T}$. We denote by $\mathbb{F}=\left\{\mathcal{F}_{t}\right\}_{0 \leq t \leq T}$ the augmentation under $\mathbb{P}$ of the natural filtration generated by the standard Brownian motion $\left\{Z_{t}\right\}_{0 \leq t \leq T}$. In what follows, (in)equalities between random variables hold $\mathbb{P}$-almost surely.

Our financial market consists of an instantaneously risk-free asset and $N$ risky stocks. We assume that trading takes place continuously. The price of the risk-free asset, $B_{t}$, obeys

$$
\frac{\mathrm{d} B_{t}}{B_{t}}=r_{t} \mathrm{~d} t, \quad B_{0}=1
$$

The scalar-valued risk-free rate process, $\left\{r_{t}\right\}_{0 \leq t \leq T}$, is $\mathcal{F}_{t}$-progressively measurable and satisfies $\int_{0}^{T}\left|r_{t}\right| \mathrm{d} t<\infty$. The $N$-dimensional vector of risky stock prices, $S_{t}$, is subject to the following equation:

$$
\frac{\mathrm{d} S_{t}}{S_{t}}=\mu_{t} \mathrm{~d} t+\sigma_{t} \mathrm{~d} Z_{t}, \quad S_{0}=\mathbf{1}_{N}
$$

with $\mathbf{1}_{N}$ denoting an $N$-dimensional vector consisting of all ones. The $N$-dimensional mean rate of return process, $\left\{\mu_{t}\right\}_{0 \leq t \leq T}$, and the $(N \times N)$-matrix-valued volatility process, $\left\{\sigma_{t}\right\}_{0 \leq t \leq T}$, are $\mathcal{F}_{t}$-progressively measurable and satisfy $\int_{0}^{T}\left\|\mu_{t}\right\| \mathrm{d} t<\infty$ and $\sum_{i=1}^{N} \sum_{j=1}^{N} \int_{0}^{T}\left(\sigma_{t}\right)_{i j}^{2} \mathrm{~d} t<\infty$, respectively ${ }^{6}$ The volatility matrix $\sigma_{t}$ satisfies the following condition for every $t \in[0, T]$ :

$$
\zeta^{\top} \sigma_{t} \sigma_{t}^{\top} \zeta \geq \epsilon\|\zeta\|^{2}, \forall \zeta \in \mathbb{R}^{N}
$$

for some $\epsilon>0$. Here, the symbol "T" denotes the transpose sign. The strong non-degeneracy condition (3) implies in particular that $\sigma_{t}$ is invertible.

\footnotetext{
${ }^{6}$ We denote by $\left(\sigma_{t}\right)_{i j}$ the $(i, j)$-element of the matrix $\sigma_{t}$.
} 
We define the $\mathcal{F}_{t}$-progressively measurable market price of risk process, $\left\{\lambda_{t}\right\}_{0 \leq t \leq T}$, as follows:

$$
\lambda_{t}=\sigma_{t}^{-1}\left(\mu_{t}-r_{t} \mathbf{1}_{N}\right)
$$

The unique positive-valued state price density process, $\left\{M_{t}\right\}_{0 \leq t \leq T}$, now satisfies (see, e.g., Karatzas and Shreve (1998)):

$$
M_{t}=\exp \left\{-\int_{0}^{t} r_{s} \mathrm{~d} s-\int_{0}^{t} \lambda_{s}^{\top} \mathrm{d} Z_{s}-\frac{1}{2} \int_{0}^{t}\left\|\lambda_{s}\right\|^{2} \mathrm{~d} s\right\}
$$

The economy consists of a single individual endowed with initial wealth $W_{0} \geq 0$. The individual seeks to maximize the total value of consumption 7 by picking an $\mathcal{F}_{t}$-progressively measurable $N$-dimensional portfolio process $\left\{\pi_{t}\right\}_{0 \leq t \leq T}$ (representing the amounts invested in the $N$ risky stocks) and an $\mathcal{F}_{t}$-progressively measurable consumption process $\left\{c_{t}\right\}_{0 \leq t \leq T}$. The portfolio and consumption processes satisfy the following integrability conditions:

$$
\int_{0}^{T} \pi_{t}^{\top} \sigma_{t} \sigma_{t}^{\top} \pi_{t} \mathrm{~d} t<\infty, \quad \int_{0}^{T}\left|\pi_{t}\left(\mu_{t}-r_{t} \mathbf{1}_{N}\right)\right| \mathrm{d} t<\infty, \quad \mathbb{E}\left[\int_{0}^{T} c_{t}^{2} \mathrm{~d} t\right]<\infty .
$$

Here, $\mathbb{E}[\cdot]$ denotes the unconditional expectation operator. The wealth process, $\left\{W_{t}\right\}_{0 \leq t \leq T}$, is subject to the following dynamic budget constraint:

$$
\mathrm{d} W_{t}=\left(r_{t} W_{t}+\pi_{t}^{\top} \sigma_{t} \lambda_{t}-c_{t}\right) \mathrm{d} t+\pi_{t}^{\top} \sigma_{t} \mathrm{~d} Z_{t}, \quad W_{0} \geq 0 \text { given }
$$

We call a consumption-portfolio strategy $\left\{c_{t}, \pi_{t}\right\}_{0 \leq t \leq T}$ admissible if the associated wealth process is uniformly bounded from below.

\footnotetext{
${ }^{7}$ Section 2.2 introduces the individual's preferences.
} 


\section{$2.2 \quad$ Preferences}

Denote by $\theta_{t}$ the individual's reference level at time $t$. Inspired by prospect theory, we assume that the general evaluation at time 0 of future gains and losses $\widehat{c}_{t}=c_{t}-\theta_{t}$ is given by

$$
V\left(\widehat{c}_{t}\right)=\int_{-\infty}^{\infty} v(x) \mathrm{d}\left[1-w_{t}\left(1-F_{\widehat{c}_{t}}(x)\right)\right]
$$

Here, $v(\cdot)$ denotes a function which evaluates realizations of $\widehat{c}_{t}$ and $w_{t}(\cdot)$ represents a function which transforms the decumulative distribution function of $\widehat{c}_{t}, 1-F_{\widehat{c}_{t}}(x)$. Note that the function $w_{t}(\cdot)$ is allowed to depend on time $t$. Intuitively, from the perspective of time 0 , the (real-world) probability that stock prices drop below a certain threshold changes with the time horizon. Hence, the individual may prefer to change the shape of the function $w_{t}(\cdot)$ as the time horizon widens.

According to prospect theory, gains and losses are treated differently. Therefore, we decompose the instantaneous utility function $v(\cdot)$ into two parts: the first part evaluates gains and the second part evaluates losses. More specifically, we write $v(\cdot)$ as follows:

$$
v\left(\widehat{c}_{t}\right)=v_{G}\left(\widehat{c}_{t}\right) \mathbb{1}_{\left[\widehat{c}_{t} \geq 0\right]}+v_{L}\left(\widehat{c}_{t}\right) \mathbb{1}_{\left[\widehat{c}_{t}<0\right]},
$$

with $v_{G}(\cdot)$ and $v_{L}(\cdot)$ denoting the instantaneous utility function for gains and the instantaneous utility function for losses, respectively.

Prospect theory states that not only gains and losses themselves but also their decumulative distribution functions are treated differently. We therefore decompose $w_{t}(\cdot)$ as follows:

$$
w_{t}\left(1-F_{\widehat{c}_{t}}\left(\widehat{c}_{t}\right)\right)=w_{t, G}\left(1-F_{\widehat{c}_{t}}\left(\widehat{c}_{t}\right)\right) \mathbb{1}_{\left[\widehat{c}_{t} \geq 0\right]}+w_{t, L}\left(1-F_{\widehat{c}_{t}}\left(\widehat{c}_{t}\right)\right) \mathbb{1}_{\left[\widehat{c}_{t}<0\right]},
$$

with $w_{t, G}(\cdot)$ and $w_{t, L}(\cdot)$ denoting the probability weighting function for gains and the probability weighting function for losses, respectively. We assume that $w_{t, G}(\cdot)$ and $w_{t, L}(\cdot)$ are strictly increasing and differentiable. Note that Tversky and Kahneman (1992) define the 
preference for a loss in a slightly different, but equivalent, way:

$$
V_{L}\left(\widehat{c}_{t}\right)=\int_{-\infty}^{0} v_{L}(x) \mathrm{d} \tilde{w}_{t, L}\left(F_{\widehat{c}_{t}}(x)\right)
$$

with $\tilde{w}_{t, L}\left(F_{\widehat{c}_{t}}(x)\right) \equiv 1-w_{t, L}\left(1-F_{\widehat{c}_{t}}(x)\right)$.

Eqn. (8) shows that the individual's preferences consist of various ingredients: the instantaneous utility function $v(\cdot)$, the function $w_{t}(\cdot)$, and the reference level $\theta_{t}$. Sections 2.2 .1 and 2.2 .2 introduce the parametric specification of the instantaneous utility function and the dynamics of the reference level, respectively. We introduce the parametric specification of the function $w_{t}(\cdot)$ in Section 3.4 .

\subsubsection{Specification of the Instantaneous Utility Function}

Following the literature on prospect theory (see, e.g., Tversky and Kahneman (1992)), we assume that

$$
\begin{gathered}
v_{G}\left(\widehat{c}_{t}\right)=\widehat{c}_{t}^{\gamma_{G}}, \\
v_{L}\left(\widehat{c}_{t}\right)=-\kappa\left(-\widehat{c}_{t}\right)^{\gamma_{L}},
\end{gathered}
$$

where $\gamma_{G} \in(0,1)$ and $\gamma_{L}>0$ are curvature parameters, and $\kappa \geq 1$ denotes the loss aversion index 8

Note that if $\gamma_{L}$ is smaller than unity, the utility function is convex-shaped below the reference level, while if $\gamma_{L}$ is larger than unity, the utility function is concave-shaped below the reference level. The utility function is concave above the reference level for all values of $\gamma_{G}$ we consider. Given $\gamma_{G}$ and $\gamma_{L}$, the loss aversion index $\kappa$ controls the steepness of the kink at the reference level.

${ }^{8}$ There is a lack of consensus in the literature on how to define loss aversion. We define loss aversion consistent with Tversky and Kahneman (1992). They define the loss aversion index (i.e., $\kappa$ ) to be the ratio between the disutility of one unit loss and the utility of one unit gain. Section 4 assumes $\gamma_{L} \geq \gamma_{G}$. Under this assumption, $-v_{L}(-x)>v_{G}(x)$ for all $x>1$, which seems reasonable in a life-cycle setting. 


\subsubsection{Reference Level Dynamics}

Motivated by the literature on internal habit formation (see, e.g., Constantinides (1990), Detemple and Zapatero (1992), and Detemple and Karatzas (2003)), we assume that the individual's reference level depends on the individual's own past consumption choices. More specifically, $\theta_{t}$ evolves as follows:

$$
\mathrm{d} \theta_{t}=\left(\beta c_{t}-\alpha \theta_{t}\right) \mathrm{d} t, \quad \theta_{0} \geq 0 \text { given. }
$$

Or, in explicit form,

$$
\theta_{t}=\beta \int_{0}^{t} \exp \{-\alpha(t-s)\} c_{s} \mathrm{~d} s+\exp \{-\alpha t\} \theta_{0} .
$$

Here, $\theta_{0}$ is the initial reference level, $\alpha \geq 0$ models the memory or persistence of the habit level, and $\beta \geq 0$ measures the importance of past consumption relative to the initial reference level ${ }^{9}$

\subsection{Optimization Problem}

The theoretical and experimental literature gives little guidance on how to apply probability weighting in an intertemporal setting, such as the one we consider. We assume that to determine lifetime utility the individual first applies prospect theory to evaluate consumption at time $t$ according to (8) yielding $V\left(c_{t}-\theta_{t}\right), t \in[0, T]$, and then aggregates in a linear manner, as follows:

$$
\mathcal{V}(c-\theta):=\int_{0}^{T} e^{-\delta t} V\left(c_{t}-\theta_{t}\right) \mathrm{d} t,
$$

with $\delta \geq 0$ denoting the subjective rate of time preference.

$\sqrt[9]{\text { Köszegi and Rabin }(2009) \text { and Pagel }(2017)}$ consider a forward-looking rather than (as in our case) backwardlooking reference level. However, many of our implications for optimal consumption behavior are consistent with theirs; see also Bilsen et al. (2019b). 
The individual faces now the following maximization problem:

$$
\begin{aligned}
\max _{c_{t}, \pi_{t}: 0 \leq t \leq T} & \int_{0}^{T} e^{-\delta t} V\left(c_{t}-\theta_{t}\right) \mathrm{d} t \\
\text { s.t. } & \mathrm{d} W_{t}=\left(r_{t} W_{t}+\pi_{t}^{\top} \sigma_{t} \lambda_{t}-c_{t}\right) \mathrm{d} t+\pi_{t}^{\top} \sigma_{t} \mathrm{~d} Z_{t}, \\
& \mathrm{~d} \theta_{t}=\left(\beta c_{t}-\alpha \theta_{t}\right) \mathrm{d} t, \quad c_{t} \geq \theta_{t}-L_{t}, \quad \text { for all } t \in[0, T],
\end{aligned}
$$

to find the optimal pre-committed consumption and portfolio strategy $\left(c_{t}^{*}, \pi_{t}^{*}\right), t \in[0, T]$. We refer to He, Strub, and Zariphopoulou (2019) for a detailed analysis of pre-committed versus time-consistent optimal portfolio strategies under probability weighting.

In (17), $L_{t} \geq 0$ denotes the maximum possible loss which is assumed to be a deterministic function of time ${ }^{10}$ We note that consumption can fall below zero, due to the randomness of the endogenous reference level. However, consumption never falls below the reference level by more than $L_{t}$. Furthermore, negative consumption is not uncommon in the consumption and portfolio choice literature (e.g., optimal consumption implied by some members of the popular HARA family can also become negative). The next section develops a solution technique to arrive at the optimal life-cycle policies.

\section{Solution Method}

The solution method consists of various steps. First, we define a dual maximization problem; see Section 3.1. Then, we transform the dual maximization problem into a problem involving the quantile function of future gains and losses; see Section 3.2 . Third, we solve this so-called quantile maximization problem by comparing, in a particular way, the optimal solutions to two separate problems; see Section 3.3 . Finally, we exploit the equivalence relationships between the dual problem and the primal problem to arrive at the optimal solution; see Section 3.4 .

\footnotetext{
${ }^{10}$ We choose the maximum possible loss $L_{t}$ such that the set of solutions to the optimization problem (17) is non-empty. We impose the condition $c_{t} \geq \theta_{t}-L_{t}$ for all $t \in[0, T]$ to guarantee that a solution always exist.
} 


\subsection{A Dual Maximization Problem}

The dynamic consumption and portfolio choice problem (17) is equivalent to the following static maximization (variational) problem (see Pliska (1986), Karatzas, Lehoczky, and Shreve (1987), and Cox and Huang (1989, 1991)):

$$
\begin{aligned}
\max _{c_{t}: 0 \leq t \leq T} & \int_{0}^{T} e^{-\delta t} V\left(c_{t}-\theta_{t}\right) \mathrm{d} t \\
\text { s.t. } & \mathbb{E}\left[\int_{0}^{T} M_{t} c_{t} \mathrm{~d} t\right] \leq W_{0}, \\
& \mathrm{~d} \theta_{t}=\left(\beta c_{t}-\alpha \theta_{t}\right) \mathrm{d} t, \quad c_{t} \geq \theta_{t}-L_{t}, \text { for all } t \in[0, T] .
\end{aligned}
$$

The optimal portfolio strategy $\pi_{t}^{*}$ follows from hedging arguments: it is determined such that it finances the optimal consumption strategy $c_{t}^{*}$.

By invoking the method used in Schroder and Skiadas (2002), we can transform the individual's maximization problem (18) into the following dual maximization problem, upon defining $\widehat{c}_{t} \equiv c_{t}-\theta_{t}$ :

$$
\begin{aligned}
\max _{\widehat{c}_{t}: 0 \leq t \leq T} & \int_{0}^{T} e^{-\delta t} V\left(\widehat{c}_{t}\right) \mathrm{d} t \\
\text { s.t. } & \mathbb{E}\left[\int_{0}^{T} \frac{\widehat{M}_{t}}{\widehat{M}_{0}} \widehat{c}_{t} \mathrm{~d} t\right] \leq \widehat{W}_{0}, \quad \widehat{c}_{t} \geq-L_{t}, \quad \text { for all } t \in[0, T] .
\end{aligned}
$$

The optimal dual portfolio choice $\widehat{\pi}_{t}^{*}$ finances the optimal dual consumption choice $\widehat{c}_{t}^{*}$. Here, the dual state price density $\widehat{M}_{t}$ and dual (or surplus) wealth $\widehat{W}_{t}$ are defined as follows:

$$
\widehat{M}_{t}=M_{t}\left(1+\beta A_{t}\right), \widehat{W}_{t}=\frac{W_{t}-A_{t} \theta_{t}}{1+\beta A_{t}}
$$

with $A_{t}$ denoting the price at time $t$ of a bond that pays the coupon process $\left\{e^{-(\alpha-\beta)(s-t)}\right\}_{s \geq t}$. 
More specifically, we have

$$
A_{t} \equiv \mathbb{E}_{t}\left[\int_{t}^{T} \frac{M_{s}}{M_{t}} \exp \{-(\alpha-\beta)(s-t)\} \mathrm{d} s\right]
$$

Furthermore, the individual's dual reference level given by

$$
\widehat{\theta}_{t}=\beta \int_{0}^{t} \exp \{-(\alpha-\beta)(t-s)\} \widehat{c}_{s} \mathrm{~d} s+\exp \{-(\alpha-\beta) t\} \theta_{0}
$$

agrees with the primal reference level in Eqn. (15), i.e., $\widehat{\theta}_{t}=\theta_{t}$ for all $t \in[0, T]$. The individual invests his dual wealth $\widehat{W}_{t}$ in a dual financial market. This dual financial market is characterized by the dual risk-free interest rate $\widehat{r}_{t}$, the dual volatility matrix $\widehat{\sigma}_{t}$, and the dual market price of risk vector $\widehat{\lambda}_{t}$ :

$$
\begin{gathered}
\widehat{r}_{t}=\beta+\frac{r_{t}-\alpha \beta A_{t}}{1+\beta A_{t}}, \quad \widehat{\sigma}_{t}=\sigma_{t}, \\
\widehat{\lambda}_{t}=\lambda_{t}-\frac{\beta}{1+\beta A_{t}} \int_{t}^{T} \exp \{-(\alpha-\beta)(s-t)\} P_{t, s} \Psi_{t, s} \mathrm{~d} s,
\end{gathered}
$$

where $P_{t, s}$ represents the price at time $t$ of a zero-coupon bond that matures at time $s \geq t$ and $\Psi_{t, s}$ denotes the volatility at time $t$ of the instantaneous return on a zero-coupon bond with maturity date $s \geq t$ (both in the primal financial market).

The next proposition follows from Schroder and Skiadas (2002).

Proposition 1. Denote by $\widehat{c}_{t}^{*}$ the optimal dual consumption choice, by $\widehat{\theta}_{t}^{*}$ the optimal dual reference level implied by substituting the optimal past dual consumption choices into 22, by $\widehat{W}_{t}^{*}$ optimal dual wealth, and by $\widehat{\pi}_{t}^{*}$ the optimal dual portfolio choice. Then:

- The optimal consumption for the individual at time $0 \leq t \leq T$ is given by

$$
c_{t}^{*}=\widehat{c}_{t}^{*}+\widehat{\theta}_{t}^{*} .
$$


- The optimal wealth for the individual at time $0 \leq t \leq T$ is given by

$$
W_{t}^{*}=\widehat{W}_{t}^{*}+\beta A_{t} \widehat{W}_{t}^{*}+A_{t} \widehat{\theta}_{t}^{*}
$$

with $A_{t}$ given by 21 .

- The optimal portfolio choice for the individual at time $0 \leq t \leq T$ is given by

$$
\pi_{t}^{*}=\widehat{\pi}_{t}^{*}+\beta A_{t} \widehat{\pi}_{t}^{*}+\left(\beta \widehat{W}_{t}^{*}+\widehat{\theta}_{t}^{*}\right)\left(\widehat{\sigma}_{t}\right)^{-1} \int_{t}^{T} \exp \{-(\alpha-\beta)(s-t)\} P_{t, s} \Psi_{t, s} \mathrm{~d} s
$$

Here, $P_{t, s}$ represents the price at time $t$ of a zero-coupon bond that matures at time $s \geq t$ and $\Psi_{t, s}$ denotes the volatility of the instantaneous return $\mathrm{d} P_{t, s} / P_{t, s}$ (both in the primal financial market).

\subsection{A Quantile Maximization Problem}

This section demonstrates how we can convert the dual maximization problem 19 into a quantile maximization problem. In the quantile maximization problem, the individual seeks to optimally choose the quantile function (i.e., inverse cumulative distribution function) of dual consumption $\widehat{c}_{t}$. After changing the individual's decision variable from dual consumption to the quantile function of dual consumption, the individual's preference measure reduces to an ordinary linear expectation. Hence, we can use conventional techniques (such as the Lagrange method) to obtain the optimal dual consumption choice. He and Zhou (2011) give a systematic account of the quantile method. We only need the following additional assumption:

Assumption 1. The dual state price density $\widehat{M}_{t}$ admits no atoms; that is, $\mathbb{P}\left\{\widehat{M}_{t}=a\right\}=0$ for all $a \in \mathbb{R}^{+}$.

Assumption 1 is satisfied if, e.g., the investment opportunity set is deterministic.

By using the substitution $z \equiv F_{\widehat{c}_{t}}(x)$, we can now transform the preference measure $V\left(\widehat{c}_{t}\right)$ 
as defined in (8) into the following ordinary (unconditional) expectation:

$$
\begin{aligned}
V\left(\widehat{c}_{t}\right) & =\int_{0}^{1} v\left(F_{\widehat{c}_{t}}^{-1}(z)\right) \mathrm{d}\left[1-w_{t}(1-z)\right]=\int_{0}^{1} v\left(F_{\widehat{c}_{t}}^{-1}(z)\right) w_{t}^{\prime}(1-z) \mathrm{d} z \\
& =\mathbb{E}\left[v\left(F_{\widehat{c}_{t}}^{-1}(Z)\right) w_{t}^{\prime}(1-Z)\right]=\mathbb{E}\left[v\left(Q_{\widehat{c}_{t}}(Z)\right) w_{t}^{\prime}(1-Z)\right] .
\end{aligned}
$$

Here, $Z$ is any uniformly distributed random variable on $[0,1]$ and $Q_{\widehat{c}_{t}}(\cdot)$ denotes the quantile function of dual consumption $\widehat{c}_{t}$.

Let us denote by $F_{\widehat{M}_{t}}(\cdot)$ the (unconditional) cumulative distribution function of the dual state price density $\widehat{M}_{t}$. By Assumption 1, we can replace the static dual budget constraint in (19) by 11

$$
\frac{1}{\widehat{M}_{0}} \mathbb{E}\left[\int_{0}^{T} Q_{\widehat{M}_{t}}\left(1-Z_{\widehat{M}_{t}}\right) Q_{\widehat{c}_{t}}\left(Z_{\widehat{M}_{t}}\right) \mathrm{d} t\right]=\widehat{W}_{0}
$$

Here, $Q_{\widehat{M}_{t}}(\cdot)$ is the quantile function of the dual state price density $\widehat{M}_{t}$ and $Z_{\widehat{M}_{t}} \equiv 1-F_{\widehat{M}_{t}}\left(\widehat{M}_{t}\right)$. Note that Eqn. (28) holds for any uniformly distributed random variable $Z$, whereas Eqn. (29) is only valid for one particular uniformly distributed random variable $Z_{\widehat{M}_{t}} \equiv 1-F_{\widehat{M}_{t}}\left(\widehat{M}_{t}\right)$.

Define $\mathcal{Q}$ to be the set of all quantile functions. The dual maximization problem 190 is

\footnotetext{
${ }^{11} \mathrm{By}$ Assumption 1, we have that $Z_{\widehat{M}_{t}}$ is a uniformly distributed random variable on $[0,1]$. Hence, $\widehat{M}_{t}=$ $Q_{\widehat{M}_{t}}\left(1-Z_{\widehat{M}_{t}}\right)$. Furthermore, $V\left(\widehat{c}_{t}^{*}\right)=V\left(Q_{\widehat{c}_{t}}^{*}\left(Z_{\widehat{M}_{t}}\right)\right)$, with $\widehat{c}_{t}^{*}$ denoting the optimal consumption choice at time $t$ and $Q_{\widehat{c}_{t}}^{*}(\cdot)$ representing its quantile function, thanks to the law-invariant nature of the individual's
} preference measure $V(\cdot)$. It now follows from Theorem B.1 in Jin and Zhou (2008) that

$$
\widehat{W}_{0} \geq \mathbb{E}\left[\int_{0}^{T} \widehat{M}_{t} \widehat{c}_{t}^{*} \mathrm{~d} t\right] \geq \mathbb{E}\left[\int_{0}^{T} \frac{\widehat{M}_{t}}{\widehat{M}_{0}} Q_{\widehat{c}_{t}}^{*}\left(Z_{\widehat{M}_{t}}\right) \mathrm{d} t\right] .
$$

If the second inequality is strict, then the individual can increase the value $V\left(\widehat{c}_{t}\right)$ as defined in 28$]$ with original initial dual wealth $\widehat{W}_{0}$, contradicting the optimality of $\widehat{c}_{t}^{*}$. Hence, at the optimum, we must have

$$
\mathbb{E}\left[\int_{0}^{T} \frac{\widehat{M}_{t}}{\widehat{M}_{0}} \widehat{c}_{t}^{*} \mathrm{~d} t\right]=\mathbb{E}\left[\int_{0}^{T} \frac{\widehat{M}_{t}}{\widehat{M}_{0}} Q_{\widehat{c}_{t}}^{*}\left(Z_{\widehat{M}_{t}}\right) \mathrm{d} t\right]=\frac{1}{\widehat{M}_{0}} \mathbb{E}\left[\int_{0}^{T} Q_{\widehat{M}_{t}}\left(1-Z_{\widehat{M}_{t}}\right) Q_{\widehat{c}_{t}}^{*}\left(Z_{\widehat{M}_{t}}\right) \mathrm{d} t\right]=\widehat{W}_{0} .
$$


equivalent to the following quantile maximization problem:

$$
\begin{aligned}
\max _{Q_{\widehat{c}_{t}}(\cdot) \in \mathcal{Q}: 0 \leq t \leq T} & \int_{0}^{T} e^{-\delta t} \mathbb{E}\left[v\left(Q_{\widehat{c}_{t}}\left(Z_{\widehat{M}_{t}}\right)\right) w_{t}^{\prime}\left(1-Z_{\widehat{M}_{t}}\right)\right] \mathrm{d} t \\
\text { s.t. } & \frac{1}{\widehat{M}_{0}} \mathbb{E}\left[\int_{0}^{T} Q_{\widehat{M}_{t}}\left(1-Z_{\widehat{M}_{t}}\right) Q_{\widehat{c}_{t}}\left(Z_{\widehat{M}_{t}}\right) \mathrm{d} t\right] \leq \widehat{W}_{0}, \\
& Q_{\widehat{c}_{t}}\left(Z_{\widehat{M}_{t}}\right) \geq-L_{t}, \text { for all } t \in[0, T] .
\end{aligned}
$$

The quantile maximization problem $(30)$ is called the quantile formulation. The individual's decision variable is the quantile function of dual consumption $Q_{\widehat{c}_{t}}(\cdot)$. Note that the preference measure in (30) arises as a special case of 28) by taking $Z=Z_{\widehat{M}_{t}}$.

The next proposition is adapted from He and Zhou (2011).

Proposition 2. Suppose that $Q_{\widehat{c}_{t}}^{*}(\cdot)$ is optimal for problem 30 . Then $\widehat{c}_{t}^{*}=Q_{\widehat{c}_{t}}^{*}\left(Z_{\widehat{M}_{t}}\right)$ is optimal for problem 19 .

\subsection{Optimal Solution}

The next step in the solution procedure is to determine the optimal quantile function of dual consumption. Proposition 3 summarizes our results. This proposition assumes that $\gamma_{L}>1$, so that the two-part power utility function $(9)$ is concave in the loss domain. Appendix B considers the case where $\gamma_{L} \leq 1$.

Proposition 3. Consider an individual with the utility function for gains 12), the utility function for losses (13), and reference level specification (15) who solves the quantile maximization problem (30). Denote by $y$ the Lagrange multiplier associated with the static budget constraint in (30), and by $\widehat{M}_{t}$ the dual state price density. Assume that $\gamma_{L}>1$. Furthermore, assume that $\quad Q_{\widehat{M}_{t}}\left(1-Z_{\widehat{M}_{t}}\right) / w_{t, G}^{\prime}\left(1-Z_{\widehat{M}_{t}}\right) \quad$ and $Q_{\widehat{M}_{t}}\left(1-Z_{\widehat{M}_{t}}\right) / w_{t, L}^{\prime}\left(1-Z_{\widehat{M}_{t}}\right)$ are non-increasing in $Z_{\widehat{M}_{t}}$ and that the equation $f_{t}\left(Z_{\widehat{M}_{t}}\right)=0$, with $f_{t}\left(Z_{\widehat{M}_{t}}\right)$ given by 40 below, has a unique solution $p_{t}^{*}$ such that $f_{t}\left(Z_{\widehat{M}_{t}}\right) \geq 0$ if and only if $Z_{\widehat{M}_{t}} \geq p_{t}^{*}$. 
Then optimal dual consumption $\widehat{c}_{t}^{*}$ is given by

$$
\widehat{c}_{t}^{*}=Q_{\widehat{c}_{t}}^{*}\left(Z_{\widehat{M}_{t}}\right)=Q_{\widehat{c}_{t, G}}^{*}\left(Z_{\widehat{M}_{t}}\right) \mathbb{1}_{\left[Z_{\widehat{M}_{t}} \geq p_{t}^{*}\right.}+Q_{\widehat{c}_{t, L}}^{*}\left(Z_{\widehat{M}_{t}}\right) \mathbb{1}_{\left[Z_{\widehat{M}_{t}}<p_{t}^{*}\right.}
$$

Here,

$$
\begin{gathered}
Q_{\widehat{c}_{t, G}}^{*}\left(Z_{\widehat{M}_{t}}\right)=\left[\frac{e^{\delta t} y Q_{\widehat{M}_{t}}\left(1-Z_{\widehat{M}_{t}}\right)}{\gamma_{G} \widehat{M}_{0} w_{t, G}^{\prime}\left(1-Z_{\widehat{M_{t}}}\right)}\right]^{\frac{1}{\gamma_{G}^{-1}}}, \\
Q_{\widehat{c}_{t, L}}^{*}\left(Z_{\widehat{M}_{t}}\right)=-\min \left\{L_{t},\left[\frac{e^{\delta t} y Q_{\widehat{M}_{t}}\left(1-Z_{\widehat{M}_{t}}\right)}{\kappa \gamma_{L} \widehat{M}_{0} w_{t, L}^{\prime}\left(1-Z_{\widehat{M}_{t}}\right)}\right]^{\frac{1}{\gamma_{L}-1}}\right\} .
\end{gathered}
$$

Proof. We first note that the assumption that $Q_{\widehat{M}_{t}}\left(1-Z_{\widehat{M}_{t}}\right) / w_{t, G}^{\prime}\left(1-Z_{\widehat{M}_{t}}\right)$ is non-increasing in $Z_{\widehat{M}_{t}}$ is similar to Assumption 4.1 in Jin and Zhou (2008).

We now adapt to the present setting the solution techniques developed by Berkelaar et al. (2004) and Basak and Shapiro (2001). The Lagrangian of the quantile maximization problem (30) is given by

$$
\begin{aligned}
\mathcal{L} & =\mathbb{E}\left[\int_{0}^{T} e^{-\delta t} v\left(Q_{\widehat{c}_{t}}\left(Z_{\widehat{M}_{t}}\right)\right) w_{t}^{\prime}\left(1-Z_{\widehat{M}_{t}}\right) \mathrm{d} t\right] \\
& -y\left(\frac{1}{\widehat{M}_{0}} \mathbb{E}\left[\int_{0}^{T} Q_{\widehat{M}_{t}}\left(1-Z_{\widehat{M}_{t}}\right) Q_{\widehat{c}_{t}}\left(Z_{\widehat{M}_{t}}\right) \mathrm{d} t\right]-\widehat{W}_{0}\right) .
\end{aligned}
$$

Here, $y$ is the Lagrange multiplier associated with the static budget constraint in 30 . The individual maximizes $e^{-\delta t} v\left(Q_{\widehat{c}_{t}}\left(Z_{\widehat{M}_{t}}\right)\right) w_{t}^{\prime}\left(1-Z_{\widehat{M}_{t}}\right)-y Q_{\widehat{M}_{t}}\left(1-Z_{\widehat{M}_{t}}\right) Q_{\widehat{c}_{t}}\left(Z_{\widehat{M}_{t}}\right) / \widehat{M}_{0}$ subject to $Q_{\widehat{c}_{t}}\left(Z_{\widehat{M}_{t}}\right) \geq-L_{t}$ for every $t$ and any state of the economy.

If the individual experiences a gain, then optimal dual consumption in the gain domain 
$Q_{\widehat{c}_{t, G}}^{*}\left(Z_{\widehat{M}_{t}}\right)$ satisfies the following first-order optimality conditions:

$$
e^{-\delta t} v_{G}^{\prime}\left(Q_{\widehat{c}_{t, G}}^{*}\left(Z_{\widehat{M}_{t}}\right)\right) w_{t, G}^{\prime}\left(1-Z_{\widehat{M}_{t}}\right)=y \frac{Q_{\widehat{M}_{t}}\left(1-Z_{\widehat{M}_{t}}\right)}{\widehat{M}_{0}}
$$

with $v_{G}(\cdot)$ given by $(12)$. Solving the optimality condition 35 , we arrive at

$$
Q_{\widehat{c}_{t, G}}^{*}\left(Z_{\widehat{M}_{t}}\right)=\left[\frac{e^{\delta t} y Q_{\widehat{M}_{t}}\left(1-Z_{\widehat{M}_{t}}\right)}{\gamma_{G} \widehat{M}_{0} w_{t, G}^{\prime}\left(1-Z_{\widehat{M}_{t}}\right)}\right]^{\frac{1}{\gamma_{G}-1}} .
$$

Because $Q_{\widehat{M}_{t}}\left(1-Z_{\widehat{M}_{t}}\right) / w_{t, G}^{\prime}\left(1-Z_{\widehat{M}_{t}}\right)$ is assumed to be non-increasing in $Z_{\widehat{M}_{t}}$, it follows that $Q_{\widehat{c}_{t, G}}^{*}(\cdot)$ is a quantile function.

If the individual experiences a loss, then optimal dual consumption in the loss domain $Q_{\widehat{c}_{t, L}}^{*}\left(Z_{\widehat{M}_{t}}\right)$ satisfies the following first-order optimality conditions:

$$
\begin{gathered}
e^{-\delta t} v_{L}^{\prime}\left(Q_{\widehat{c}_{t, L}}^{*}\left(Z_{\widehat{M}_{t}}\right)\right) w_{t, L}^{\prime}\left(1-Z_{\widehat{M}_{t}}\right)=y \frac{Q_{\widehat{M}_{t}}\left(1-Z_{\widehat{M}_{t}}\right)}{\widehat{M}_{0}}-x_{t}, \quad Q_{\widehat{c}_{t, L}}^{*}\left(Z_{\widehat{M}_{t}}\right) \geq-L_{t}, \\
x_{t}\left(Q_{\widehat{c}_{t, L}^{*}}\left(Z_{\widehat{M}_{t}}\right)+L_{t}\right)=0, \quad x_{t} \geq 0
\end{gathered}
$$

with $v_{L}(\cdot)$ given by $(13)$ and $x_{t}$ denoting the Lagrange multiplier associated with the constraint on dual consumption. Solving the optimality conditions $(37)-(38)$, we arrive at

$$
Q_{\widehat{c}_{t, L}}^{*}\left(Z_{\widehat{M}_{t}}\right)=-\min \left\{L_{t},\left[\frac{e^{\delta t} y Q_{\widehat{M}_{t}}\left(1-Z_{\widehat{M}_{t}}\right)}{\kappa \gamma_{L} \widehat{M}_{0} w_{t, L}^{\prime}\left(1-Z_{\widehat{M}_{t}}\right)}\right]^{\frac{1}{\gamma_{L}-1}}\right\} .
$$

Because $Q_{\widehat{M}_{t}}\left(1-Z_{\widehat{M}_{t}}\right) / w_{t, L}^{\prime}\left(1-Z_{\widehat{M}_{t}}\right)$ is assumed to be non-increasing in $Z_{\widehat{M}_{t}}$, it follows that $Q_{\widehat{c}_{t, L}}^{*}(\cdot)$ is a quantile function.

To determine whether the individual experiences a gain or a loss, we introduce the following 
function:

$$
\begin{aligned}
f_{t}\left(Z_{\widehat{M}_{t}}\right)= & e^{-\delta t} v_{G}\left(Q_{\widehat{c}_{t, G}}^{*}\left(Z_{\widehat{M}_{t}}\right)\right) w_{t, G}^{\prime}\left(1-Z_{\widehat{M}_{t}}\right)-y \frac{Q_{\widehat{M}_{t}}\left(1-Z_{\widehat{M}_{t}}\right)}{\widehat{M}_{0}} Q_{\widehat{c}_{t, G}}^{*}\left(Z_{\widehat{M}_{t}}\right) \\
& -\left(e^{-\delta t} v_{L}\left(Q_{\widehat{c}_{t, L}}^{*}\left(Z_{\widehat{M}_{t}}\right)\right) w_{t, L}^{\prime}\left(1-Z_{\widehat{M}_{t}}\right)-y \frac{Q_{\widehat{M}_{t}}\left(1-Z_{\widehat{M}_{t}}\right)}{\widehat{M}_{0}} Q_{\widehat{c}_{t, L}}^{*}\left(Z_{\widehat{M}_{t}}\right)\right) .
\end{aligned}
$$

Optimal dual consumption $Q_{\widehat{c}_{t}}^{*}\left(Z_{\widehat{M}_{t}}\right)$ is equal to $Q_{\widehat{c}_{t, G}}^{*}\left(Z_{\widehat{M}_{t}}\right)$ if $f_{t}\left(Z_{\widehat{M}_{t}}\right) \geq 0$; and equals $Q_{\widehat{c}_{t, L}}^{*}\left(Z_{\widehat{M}_{t}}\right)$ otherwise. Because, by assumption, the equation $f_{t}\left(Z_{\widehat{M}_{t}}\right)=0$ has a unique solution $p_{t}^{*}$ such that $f_{t}\left(Z_{\widehat{M}_{t}}\right) \geq 0$ if and only if $Z_{\widehat{M}_{t}} \geq p_{t}^{*}$, it follows that $Q_{\widehat{c}_{t}}^{*}(\cdot)$ is a quantile function.

A standard verification (see Karatzas and Shreve (1998), p. 103) shows that the optimal solution obtained from the Lagrangians equals the optimal solution of the quantile problem.

We note that even without the requirement that $Q_{\widehat{M}_{t}}\left(1-Z_{\widehat{M}_{t}}\right) / w_{t, G}^{\prime}\left(1-Z_{\widehat{M}_{t}}\right)$ and $Q_{\widehat{M}_{t}}\left(1-Z_{\widehat{M}_{t}}\right) / w_{t, L}^{\prime}\left(1-Z_{\widehat{M}_{t}}\right)$ are non-increasing in $Z_{\widehat{M}_{t}}$, the quantile maximization problem (30) can still be solved; see He and Zhou (2016) and Xia and Zhou (2016) for more details on this subject matter.

We observe from (32), (33) and (40) that optimal dual consumption depends to a large extent on the specification of the probability weighting function $w_{t}(\cdot)$. Section 3.4 introduces a parametric specification of the probability weighting function for which we are able to compute optimal consumption in closed-form.

\subsection{Analytical Expression of Optimal Consumption}

This section presents the optimal consumption choice in closed-form. To arrive at closedform expressions for the optimal life-cycle policies, we make the following assumption:

Assumption 2. The state price density $M_{t}$ is log-normally distributed for every $t \in[0, T]$.

Furthermore, inspired by the so-called Esscher-Girsanov transform of Goovaerts and Laeven (2008) (see also He and Zhou (2016), Jin and Zhou (2008), Labuschagne and Offwood (2010), 
and Wang (2000)), we assume that the probability weighting functions $w_{t, G}(\cdot)$ and $w_{t, L}(\cdot)$ satisfy:

$$
\begin{aligned}
& w_{t, G}^{\prime}(p)= \begin{cases}k_{t, G} \cdot\left[Q_{\widehat{M}_{t}}(p)\right]^{a_{G}}, & \text { if } p \leq \bar{p}_{G}, \\
k_{t, G} \cdot\left[Q_{\widehat{M}_{t}}\left(\bar{p}_{G}\right)\right]^{a_{G}-b_{G}} \cdot\left[Q_{\widehat{M}_{t}}(p)\right]^{b_{G}}, & \text { if } p>\bar{p}_{G},\end{cases} \\
& w_{t, L}^{\prime}(p)= \begin{cases}k_{t, L} \cdot\left[Q_{\widehat{M}_{t}}(p)\right]^{a_{L}}, & \text { if } p \leq \bar{p}_{L}, \\
k_{t, L} \cdot\left[Q_{\widehat{M}_{t}}\left(\bar{p}_{L}\right)\right]^{a_{L}-b_{L}} \cdot\left[Q_{\widehat{M}_{t}}(p)\right]^{b_{L}}, & \text { if } p>\bar{p}_{L} .\end{cases}
\end{aligned}
$$

Here, $a_{G} \leq 0, a_{L} \leq 0,0 \leq b_{G} \leq 1,0 \leq b_{L} \leq 1, \bar{p}_{G}>0$ and $\bar{p}_{L}>0$ are preference parameters. The analytical expressions for $w_{t, G}(\cdot)$ and $w_{t, L}(\cdot)$ are given in Appendix $\mathrm{A}$ which also defines $k_{t, G}$ and $k_{t, L}$. We call the parameters $\bar{p}_{G}$ and $\bar{p}_{L}$ the inflection points ${ }^{12}$ The probability weighting function $w_{t, G}(\cdot)$ is concave up to $\bar{p}_{G}$, and convex beyond $\bar{p}_{G}$, and similarly for $w_{t, L}(\cdot)$. It follows that the shape parameter $a_{G}$ controls the degree of concavity in the domain $0 \leq p \leq \bar{p}_{G}$. More specifically, the smaller $a_{G}$ is, the more concave the weighting function $w_{t, G}(\cdot)$ will be. The shape parameter $b_{G}$ models the degree of convexity in the domain $\bar{p}_{G} \leq p \leq 1$. A larger value of $b_{G}$ corresponds to a more convex probability weighting function. Figure 1 illustrates the probability weighting function $w_{t, G}(\cdot)$ for various sets of parameter values. The figure shows that the probability weighting function $w_{t, G}(\cdot)$ is allowed to be inverse S-shaped consistent with prospect theory.

\section{[Place Figure 1 about here]}

We summarize the optimal consumption choice in the following theorem.

Theorem 1. Consider an individual with the utility function for gains (12), the utility function for losses (13), reference level specification (15), the probability weighting function for gains satisfying (41) and the probability weighting function for losses satisfying (42) who solves the optimization problem (18). Denote by $\theta_{t}^{*}$ the individual's optimal reference level at time $t$ implied by substituting the optimal past consumption choices into (15), by y the Lagrange multiplier associated with the static budget constraint in 18, and by $\widehat{M}_{t}$ the dual state price density.

\footnotetext{
${ }^{12}$ The literature finds that $\bar{p}_{G}$ and $\bar{p}_{L}$ are about $1 / 3$ (see, e.g., Wu and Gonzalez (1996) and Abdellaoui (2000)).
} 
Assume that $\gamma_{L}>1$ and $b_{G} \leq \gamma_{G}$. Then the optimal consumption choice at time $t$ is given by

$$
c_{t}^{*}=\theta_{t}^{*}+\widehat{c}_{t}^{*},
$$

with

$$
\widehat{c}_{t}^{*}=Q_{\widehat{c}_{t, G}}^{*}\left(1-F_{\widehat{M}_{t}}\left(\widehat{M}_{t}\right)\right) \mathbb{1}_{\left[\widehat{M}_{t} \leq \xi_{t}\right]}+Q_{\widehat{c}_{t, L}}^{*}\left(1-F_{\widehat{M}_{t}}\left(\widehat{M}_{t}\right)\right) \mathbb{1}_{\left[\widehat{M}_{t}>\xi_{t}\right]}
$$

Here, $\xi_{t} \equiv Q_{\widehat{M}_{t}}\left(1-p_{t}^{*}\right)$ and

$$
\begin{gathered}
Q_{\widehat{c}_{t, G}}^{*}\left(1-F_{\widehat{M}_{t}}\left(\widehat{M}_{t}\right)\right)= \begin{cases}d_{t, 1}\left(\widehat{M}_{t}\right)^{\frac{1-a_{G}}{\gamma_{G}-1}}, & \text { if } \widehat{M}_{t} \leq Q_{\widehat{M}_{t}}\left(\bar{p}_{G}\right), \\
d_{t, 2}\left(\widehat{M}_{t}\right)^{\frac{1-b_{G}}{\gamma_{G}-1}}, & \text { if } \widehat{M}_{t}>Q_{\widehat{M}_{t}}\left(\bar{p}_{G}\right),\end{cases} \\
Q_{\widehat{c}_{t, L}}^{*}\left(1-F_{\widehat{M}_{t}}\left(\widehat{M}_{t}\right)\right)= \begin{cases}-\min \left\{L_{t}, d_{t, 3}\left(\widehat{M}_{t}\right)^{\frac{1-a_{L}}{\gamma_{L}-1}}\right\}, & \text { if } \widehat{M}_{t} \leq Q_{\widehat{M}_{t}}\left(\bar{p}_{L}\right), \\
-\min \left\{L_{t}, d_{t, 4}\left(\widehat{M}_{t}\right)^{\frac{1-b_{L}}{\gamma_{L}-1}}\right\}, & \text { if } \widehat{M}_{t}>Q_{\widehat{M}_{t}}\left(\bar{p}_{L}\right),\end{cases}
\end{gathered}
$$

where

$$
\begin{aligned}
& d_{t, 1}=\left(\frac{y e^{\delta t}}{k_{t, G} \gamma_{G} \widehat{M}_{0}}\right)^{\frac{1}{\gamma_{G}-1}}, \quad d_{t, 2}=\left(\frac{y e^{\delta t}}{k_{t, G}\left[Q_{\widehat{M}_{t}}\left(\bar{p}_{G}\right)\right]^{a_{G}-b_{G}} \gamma_{G} \widehat{M}_{0}}\right)^{\frac{1}{\gamma_{G}-1}} \\
& d_{t, 3}=\left(\frac{y e^{\delta t}}{k_{t, L} \kappa \gamma_{L} \widehat{M}_{0}}\right)^{\frac{1}{\gamma_{L}-1}}, d_{t, 4}=\left(\frac{y e^{\delta t}}{k_{t, L}\left[Q_{\widehat{M}_{t}}\left(\bar{p}_{L}\right)\right]^{a_{L}-b_{L}} \kappa \gamma_{L} \widehat{M}_{0}}\right)^{\frac{1}{\gamma_{L}-1}}
\end{aligned}
$$

The threshold $p_{t}^{*}$ is the solution to the equation $f_{t}\left(Z_{\widehat{M}_{t}}\right)=0$ with $f_{t}\left(Z_{\widehat{M}_{t}}\right)$ given by 40 above. We choose $y$ such that the static budget constraint in (18) holds with equality.

Proof. By substituting the expression for $w_{t, G}^{\prime}(\cdot)$ (see 411) ) and the expression for $w_{t, L}^{\prime}(\cdot)$ (see (42) into (32) and (33) and using $Z_{\widehat{M}_{t}} \equiv 1-F_{\widehat{M}_{t}}\left(\widehat{M}_{t}\right)$ and $Q_{\widehat{M}_{t}}\left(F_{\widehat{M}_{t}}\left(\widehat{M}_{t}\right)\right)=\widehat{M}_{t}$, we obtain (45) and (46), which are clearly decreasing in $\widehat{M}_{t}$. Furthermore, we find that, under the condition $b_{G} \leq \gamma_{G}, \lim _{Z_{\widehat{M}_{t}} \rightarrow 1} f_{t}\left(Z_{\widehat{M}_{t}}\right)=+\infty, \lim _{Z_{\widehat{M}_{t}} \rightarrow 0}\left(Z_{\widehat{M}_{t}}\right)=-\infty$ and $f_{t}^{\prime}\left(Z_{\widehat{M}_{t}}\right)>0$ for all $Z_{\widehat{M}_{t}}$. Hence, the equation $f_{t}\left(Z_{\widehat{M}_{t}}\right)=0$ has one unique solution $p_{t}^{*}$ such that $f_{t}\left(Z_{\widehat{M}_{t}}\right) \geq 0$ 
if and only if $Z_{\widehat{M}_{t}} \geq p_{t}^{*}$. As a result, $Q_{\widehat{c}_{t}}^{*}\left(1-F_{\widehat{M}_{t}}\left(\widehat{M}_{t}\right)\right)$ is equal to $Q_{\widehat{c}_{t, G}}^{*}\left(1-F_{\widehat{M}_{t}}\left(\widehat{M}_{t}\right)\right)$ if $\widehat{M}_{t} \leq Q_{\widehat{M}_{t}}\left(1-p_{t}^{*}\right)$; and equals $Q_{\widehat{c}_{t, L}}^{*}\left(1-F_{\widehat{M}_{t}}\left(\widehat{M}_{t}\right)\right)$ otherwise. By Propositions 2 and 3 $\widehat{c}_{t}^{*}=Q_{\widehat{c}_{t, G}}^{*}\left(1-F_{\widehat{M}_{t}}\left(\widehat{M}_{t}\right)\right) \mathbb{1}_{\left[\widehat{M}_{t} \leq Q_{\widehat{M}_{t}}\left(1-p_{t}^{*}\right)\right]}+Q_{\widehat{c}_{t, L}}^{*}\left(1-F_{\widehat{M}_{t}}\left(\widehat{M}_{t}\right)\right) \mathbb{1}_{\left[\widehat{M}_{t}>Q_{\widehat{M}_{t}}\left(1-p_{t}^{*}\right)\right]} \cdot \quad$ The optimal consumption choice $c_{t}^{*}$ finally follows from the equivalence relationships between the dual problem and the primal (original) problem summarized in Proposition 1.

\section{Analysis of the Optimal Life-Cycle Policies}

\subsection{Benchmark Parameter Values}

In the analysis that follows we make the following assumptions. The individual invests his wealth in a risk-free asset and a single risky stock. We assume a constant investment opportunity set. That is, $r_{t}=r, \sigma_{t}=\sigma$ and $\lambda_{t}=\lambda$ for every $t$. The equity risk premium $\sigma \lambda=\mu-r$ is set at $4 \%$, the risk-free rate $r$ at $1 \%$, and the stock return volatility $\sigma$ at $20 \%$. These estimates are the same as those used by Gomes, Kotlikoff, and Viceira (2008). The terminal time $T$ is set equal to 20 , the initial reference level $\theta_{0}$ to $\$ 50 \mathrm{k}$ the curvature parameter for losses $\gamma_{L}$ to 1.3 , the curvature parameter for gains $\gamma_{G}$ to 0.4 , the subjective rate of time preference $\delta$ to $4 \%,{ }^{14}$ and the loss aversion index $\kappa$ to 2 . The literature reports that estimates of the (median) loss aversion index range from 1 to 5 (see, e.g., Abdellaoui, Bleichrodt, and L'Haridon (2008)). We set the individual's initial wealth $W_{0}$ equal to the price of a fixed annuity with payment $\theta_{0}=\$ 50 \mathrm{k}$. That is,

$$
W_{0}=\theta_{0} \int_{0}^{T} \exp \{-r t\} \mathrm{d} t
$$

The parameters $\alpha$ and $\beta$ are set equal to zero. The reference level is thus constant over time 15 Section 4.4 relaxes this parameter restriction.

\footnotetext{
${ }^{13}$ This approximately corresponds to the median household income in the U.S. in 2014 .

${ }^{14}$ Samwick (1998) finds that time preference rates for U.S. households are between $3 \%$ and $4 \%$.

${ }^{15}$ Bilsen et al. (2019b) and Pagel (2017) extensively analyze the impact of an endogenous reference level on the optimal life-cycle policies.
} 


\subsection{Optimal Consumption Choice}

Theorem 1 shows that the individual divides the states of the economy into four categories: very good scenarios, i.e., $\widehat{M}_{t} \leq \min \left\{Q_{\widehat{M}_{t}}\left(\bar{p}_{G}\right), \xi_{t}\right\}$, good scenarios, i.e., $Q_{\widehat{M}_{t}}\left(\bar{p}_{G}\right)<\widehat{M}_{t} \leq \xi_{t}$, bad scenarios, i.e., $\xi_{t}<\widehat{M}_{t} \leq Q_{\widehat{M}_{t}}\left(\bar{p}_{L}\right)$, and very bad scenarios, i.e., $\widehat{M}_{t}>\max \left\{Q_{\widehat{M}_{t}}\left(\bar{p}_{L}\right), \xi_{t}\right\}$. In very good and good scenarios, consumption is larger than the reference level, while in bad and very bad scenarios, consumption is smaller than the reference level. Figure 2 shows the optimal consumption choice of the individual at time $t=10$ as a function of the log dual pricing kernel for various sets of parameter values (note that in the absence of the endogeneity of the reference level, the dual state price density coincides with the primal state price density). The dasheddotted lines represent the optimal consumption strategy of an individual with CRRA utility (Merton (1969)). We observe from Theorem 1 and Figure 2 that the curvature parameters $a_{G}$, $b_{G}, a_{L}$ and $b_{L}$ determine for a large part the sensitivity of optimal consumption to economic shocks.

The reference level is another important factor that affects the sensitivity of optimal consumption to economic shocks. In particular, in scenarios where optimal consumption slightly exceeds the reference level, optimal consumption is relatively insensitive to economic shocks; see Figure 2(a), Indeed, in these scenarios, the individual implements a conservative investment strategy so as to avoid that future optimal consumption levels drop below the reference level; see Bilsen et al. (2019b) for an extensive analysis.

The empirical literature suggests that $\bar{p}_{G}$ is (approximately) equal to $\bar{p}_{L}$; see, e.g., $\mathrm{Wu}$ and Gonzalez (1996). Under this parameter restriction, the individual divides the state of the economy into three (instead of four) categories. With this restriction imposed, we can now distinguish between two cases. In the first case, $Q_{\widehat{M}_{t}}\left(\bar{p}_{G}\right)=Q_{\widehat{M}_{t}}\left(\bar{p}_{L}\right)$ is smaller than the threshold $\xi_{t}$. It follows from Theorem 1 that if $Q_{\widehat{M}_{t}}\left(\bar{p}_{G}\right) \leq \xi_{t}$, then the curvature parameter $a_{L}$ does not play a role. Hence, in this case only the curvature parameters $a_{G}, b_{G}$ and $b_{L}$ control the sensitivity of optimal consumption to economic shocks. Figure 2 assumes $\bar{p}_{G}=\bar{p}_{L}=1 / 3$, which is consistent with empirical estimates (see, e.g., Wu and Gonzalez (1996) and Abdellaoui $(2000)$ ). For every set of parameter values we consider, it now holds that $Q_{\widehat{M}_{t}}\left(\bar{p}_{G}\right) \leq \xi_{t}$. The 
probability of a gain is thus larger than $\bar{p}_{G}=1 / 3$. The second case assumes that $Q_{\widehat{M}_{t}}\left(\bar{p}_{G}\right)$ exceeds the threshold $\xi_{t}$ (i.e., the probability of a gain is smaller than $\bar{p}_{G}=1 / 3$ ). In the numerical illustrations, we do not consider this case.

We find that as the degree of overweighting of unlikely unfavorable events increases (i.e., $b_{G} \in\left[0, \gamma_{G}\right]$ and $b_{L} \in[0,1]$ go up), the individual demands more protection against downside risk. Optimal consumption thus becomes less sensitive to economic shocks; see Figure 2(b). Intuitively, the larger the extent to which probabilities of unlikely unfavorable events are overweighted, the lower the individual's willingness to invest in the stock market, and hence the smaller the sensitivity of current optimal consumption to economic shocks will be. In particular, if the curvature parameter $b_{L}$ equals the maximum value (i.e., $b_{L}=1$ ), then the individual consumes $c_{t}=\theta_{t}-d_{4, t}$ in scenarios where the dual pricing kernel $\widehat{M}_{t}$ is larger than the threshold $\xi_{t}$; see Figure 2(1). In these scenarios, consumption does not depend on the dual pricing kernel at all. We note that He and Zhou (2016) already show that in a terminal wealth setting with a globally concave utility function, overweighting of unlikely unfavorable events may lead to portfolio insurance strategies. We show that portfolio insurance may arise in a setting with intertemporal consumption choice.

Finally, we find that as the degree of overweighting of unlikely favorable events increases (i.e., $a_{G} \in(-\infty, 0]$ goes down), optimal consumption becomes more sensitive to economic shocks; see Figure 2(d), Intuitively, the larger the extent to which probabilities of unlikely favorable events are overweighted, the higher the individual's willingness to invest in the stock market, and hence the larger the sensitivity of current optimal consumption to economic shocks will be.

\section{[Place Figure 2 about here]}

\subsection{Optimal Portfolio Choice}

Using the principle of hedging, we find that the optimal dual portfolio choice $\widehat{\pi}_{t}^{*}$ at time $t$ is given by

$$
\widehat{\pi}_{t}^{*}=-\frac{\partial \widehat{W}_{t}^{*}}{\partial \widehat{M}_{t}} \frac{\widehat{\lambda}_{t}}{\widehat{\sigma}_{t}} .
$$


Here, $\widehat{W}_{t}^{*}$ denotes the optimal dual wealth level at time $t$. The optimal portfolio choice $\pi_{t}^{*}$ follows from Proposition 1. Figure 3 illustrates the share of wealth invested in the risky stock at time $t=10$ as a function of the log dual pricing kernel for various sets of parameter values. The figure shows that if consumption is slightly above the reference level, the share of wealth invested in the risky stock is relatively low; see Figure 3)(a). Indeed, in these scenarios, the individual wants to avoid that future consumption levels drop below the reference level. As a result, he implements a relatively conservative portfolio strategy.

Figure 3 assumes $\bar{p}_{G}=\bar{p}_{L}=1 / 3$. This assumption implies that for every set of parameter values we explore, $a_{L}$ does not play a role. Figures $3\left[(\mathrm{~b})\right.$ and $3\left[(\mathrm{c})\right.$ show that if $b_{G}$ and $b_{L}$ are larger than zero, then the share of wealth invested in the risky stock is relatively low. Indeed, if the individual overweights probabilities of unlikely unfavorable events, he is not willing to invest heavily in the stock market.

Finally, Figure 3(d) considers the impact of $a_{G}$ on the optimal portfolio strategy. We observe that if $a_{G}<0$, the individual implements a relatively risky investment strategy. Indeed, if the individual overweights probabilities of unlikely favorable events, he has a strong preference to invest a large part of his wealth in the stock market.

\section{[Place Figure 3 about here]}

\subsection{Welfare Analysis}

This section explores the welfare costs associated with incorrectly assuming CRRA utility. More specifically, we consider an individual with prospect theory preferences who delegates his (dis)saving and portfolio decisions to a professional asset manager (e.g., pension fund). The asset manager incorrectly assumes that all his clients behave in accordance with CRRA utility. He thus makes (dis)saving and portfolio decisions on behalf of his clients based on CRRA utility. The only decision a client has to make is choosing the preferred value of the relative risk aversion coefficient. This coefficient completely characterizes the CRRA portfolio strategy. We assume that our individual with prospect theory preferences chooses the value of the relative risk aversion coefficient in such a way that the difference between his actual utility (i.e., the 
utility level corresponding to the suboptimal CRRA life-cycle strategies) and his optimal utility (i.e., the utility level corresponding to the optimal prospect theory life-cycle strategies) is as small as possible.

Table 1 reports the minimum welfare losses due to loss aversion only. We measure minimum welfare losses in terms of the relative decline in certainty equivalent consumption. ${ }^{16}$ The number between brackets denotes the value of the relative risk aversion coefficient that minimizes the difference between the individual's actual utility and the individual's optimal utility. For the benchmark parameter values (see Section 4.1), we find that the minimum welfare loss equals $0.28 \%$. This loss is achieved by choosing the relative risk aversion coefficient equal to 40.9 . We observe that an increase in $\gamma_{G}$ and a decrease in $\gamma_{L}$ lead to a higher minimum welfare loss: it becomes more difficult to mimic the optimal prospect theory life-cycle policies with the CRRA life-cycle policies. Also, the minimum welfare loss is a decreasing function of the loss aversion index $\kappa$. Indeed, in the limiting case where $\kappa$ equals infinity, the minimum welfare loss vanishes (i.e., both the optimal and the CRRA portfolio strategy reduce to the risk-free portfolio strategy).

[Place Table 1 about here]

Table 2 reports the minimum welfare losses due to both loss aversion and endogenous updating of the reference level. We observe that the minimum welfare loss increases at an increasing rate as the degree of endogeneity $\beta$ rises. Hence, an individual whose reference level is very sensitive to his own past consumption choices (i.e., high value for $\beta$ ) incurs a relatively large minimum welfare loss. Indeed, under endogenous updating of the reference level, consumption exhibits excess smoothness (i.e., consumption under-responds to financial shocks) and excess sensitivity (past financial shocks have predictive power for current and future consumption growth), while under CRRA utility, financial shocks are directly reflected into current consumption; see Bilsen et al. (2019b).

\section{[Place Table 2 about here]}

\footnotetext{
${ }^{16}$ We define the certainty equivalent of an uncertain consumption strategy to be the certain consumption level that yields indifference to the uncertain consumption strategy.
} 
Table 3 reports the minimum welfare losses due to both loss aversion and probability weighting. We observe that if the individual overweights probabilities of unlikely unfavorable events (i.e., $b_{G}$ and $b_{L}$ are positive), the minimum welfare loss is very low. Indeed, in that case, a very conservative CRRA portfolio strategy almost perfectly mimics the optimal prospect theory portfolio strategy. However, if the individual overweights probabilities of unlikely favorable events (i.e., $a_{G}$ is negative), the minimum welfare loss is larger: it is more difficult to mimic the optimal prospect theory life-cycle policies with the CRRA life-cycle policies.

\section{[Place Table 3 about here]}

Finally, Table 4 reports the minimum welfare losses due to the joint impact of loss aversion, endogenous updating of the reference level and probability weighting. We observe that an endogenous reference level causes the minimum welfare losses to increase substantially. Furthermore, consistent with Table 3 , a more negative $a_{G}$ leads to a higher minimum welfare loss, while a larger $b_{G}$ and $b_{L}$ lead to a lower minimum welfare loss.

\section{[Place Table 4 about here]}

Our computations thus reveal that the minimum welfare loss can be substantial (i.e., more than $10 \%$ ) depending on the values of the individual's preference parameters.

\subsection{Implications for Annuity Product Design}

Our findings have important implications for the optimal design of investment-linked annuity products. If aiming to comply with prospect theory preferences, an annuity provider should offer an investment-linked annuity product with the following features. First, due to loss aversion, the annuity payout ratio (i.e., consumption-to-wealth ratio) should, in most economic states, increase following a stock price decline: an individual with prospect theory preferences has a strong preference to protect current consumption. This feature implies that the portfolio strategy should be very conservative as long as annuity wealth is just sufficient to finance future reference levels. In either a stock market boom or a stock market bust, the portfolio strategy 
should be less conservative. Intuitively, in periods of bust, a provider should implement a relatively risky portfolio strategy in the hope to recoup previous losses, while in periods of boom, a relatively risky portfolio strategy is not likely to lead to a loss with respect to the reference level in the near future.

Second, due to the endogeneity of the reference level, an annuity provider should delay reductions in the current annuity payment following a drop in annuity wealth. ${ }^{17}$ Indeed, with a decreasing endogenous reference level, a reduction in a future annuity payment is less painful than a reduction in a current annuity payment. A consequence of this feature is that the annuity provider should, on average, decrease the share of annuity wealth invested in the risky stock as the individual ages. Intuitively, as the individual gets older, the available time to absorb wealth shocks decreases. To be able to provide an excessively smooth payment stream at high ages, the annuity provider should thus invest less in risky stocks as the date of death approaches.

Third, if individuals overweight unlikely unfavorable events, then the annuity payment should be rather insensitive to economic shocks. If individuals sufficiently overweight unlikely unfavorable events, the annuity product should be sold with a guaranteed minimum income benefit (GMIB). As a result, the annuity provider should implement a very conservative portfolio strategy in case of overweighting of unlikely unfavorable events. It could be even the case that the annuity provider should, most of the time, not invest in the stock market at all.

Finally, if individuals overweight unlikely favorable events, then economic shocks should have a relatively large impact on the annuity payment. Intuitively, if an individual perceives the probability of an unlikely favorable event to be relatively large, he prefers to take a relatively risky position in the stock market. In case both unlikely favorable and unlikely unfavorable events are overweighted, the annuity provider should invest aggressively in good economic times and conservatively in bad economic times.

\footnotetext{
${ }^{17}$ Some existing annuity products already delay reductions in current payouts following wealth shocks. For more details about existing annuity products incorporating this feature, see, e.g., Guillén, Jørgensen, and Nielsen (2006), Jørgensen and Linnemann(2011), Guillén, Nielsen, Pérez-Marín, and Petersen (2013), Linnemann, Bruhn, and Steffensen (2014), and Maurer, Mitchell, Rogalla, and Siegelin (2016).
} 


\section{Conclusion}

We have explored the dynamic consumption and portfolio choice of an individual with prospect theory preferences. Our individual is loss averse, endogenously updates his reference level over time, and distorts probabilities.

To arrive at closed-form expressions, we have applied four steps. First, we have transformed the maximization problem into a dual maximization problem. Second, we have rewritten the dual problem in terms of the quantile function of future gains and losses. Third, we have solved the quantile maximization problem. Finally, we have exploited the equivalence relationships between the dual problem and the primal (original) problem to obtain the optimal solution.

We have shown that the optimal consumption strategy is rather insensitive to shocks. In particular, if our individual sufficiently overweights unlikely unfavorable events, our model generates an endogenous floor on consumption. Probability weighting may thus justify why investment-linked annuities are often sold with a guaranteed minimum income benefit. Also, we have shown that a person with prospect theory preferences typically follows a conservative portfolio strategy. In case the individual sufficiently overweights unlikely favorable events, he implements a substantially riskier portfolio strategy, especially in good scenarios.

Our closed-form solutions are obtained for canonical parametric specifications of the instantaneous utility functions, the reference level dynamics, the probability weighting functions, and the state price density. In future work, one may endeavor exploring extensions of each of these elements. 


\section{A Specifications of Probability Weighting Functions}

This appendix specifies the probability weighting function for gains $w_{t, G}(\cdot)$ and the probability weighting function for losses $w_{t, L}(\cdot)$. Let $\log X_{t} \sim \mathcal{N}\left(\bar{\mu}_{t}, \bar{\sigma}_{t}\right)$ with $\Phi(\cdot)$ denoting the standard normal CDF. Then:

$$
\begin{aligned}
& w_{t, G}(p)= \begin{cases}k_{t, G} \cdot f_{t, G} \cdot \Phi\left(\Phi^{-1}(p)-a_{G} \bar{\sigma}_{t}\right), & \text { if } p \in\left[0, \bar{p}_{G}\right], \\
k_{t, G} \cdot\left(\left[Q_{X_{t}}\left(\bar{p}_{G}\right)\right]^{a_{G}-b_{G}} \cdot g_{t, G} \cdot \Phi\left(\Phi^{-1}(p)-b_{G} \bar{\sigma}_{t}\right)+h_{t, G}\right), & \text { if } p \in\left[\bar{p}_{G}, 1\right] .\end{cases} \\
& w_{t, L}(p)= \begin{cases}k_{t, L} \cdot f_{t, L} \cdot \Phi\left(\Phi^{-1}(p)-a_{L} \bar{\sigma}_{t}\right), & \text { if } p \in\left[0, \bar{p}_{L}\right], \\
k_{t, L} \cdot\left(\left[Q_{X_{t}}\left(\bar{p}_{L}\right)\right]^{a_{L}-b_{L}} \cdot g_{t, L} \cdot \Phi\left(\Phi^{-1}(p)-b_{L} \bar{\sigma}_{t}\right)+h_{t, L}\right), & \text { if } p \in\left[\bar{p}_{L}, 1\right] .\end{cases}
\end{aligned}
$$

Here,

$$
\begin{gathered}
f_{t, G}=\exp \left\{a_{G} \bar{\mu}_{t}+\frac{1}{2} a_{G}^{2} \bar{\sigma}_{t}^{2}\right\}, \quad f_{t, L}=\exp \left\{a_{L} \bar{\mu}_{t}+\frac{1}{2} a_{L}^{2} \bar{\sigma}_{t}^{2}\right\}, \\
g_{t, G}=\exp \left\{b_{G} \bar{\mu}_{t}+\frac{1}{2} b_{G}^{2} \bar{\sigma}_{t}^{2}\right\}, \quad g_{t, L}=\exp \left\{b_{L} \bar{\mu}_{t}+\frac{1}{2} b_{L}^{2} \bar{\sigma}_{t}^{2}\right\}, \\
h_{t, G}=\Phi\left(\Phi^{-1}\left(\bar{p}_{G}\right)-a_{G} \bar{\sigma}_{t}\right) f_{t, G}-\Phi\left(\Phi^{-1}\left(\bar{p}_{G}\right)-b_{G} \bar{\sigma}_{t}\right)\left[Q_{X_{t}}\left(\bar{p}_{G}\right)\right]^{a_{G}-b_{G}} g_{t, G}, \\
h_{t, L}=\Phi\left(\Phi^{-1}\left(\bar{p}_{L}\right)-a_{L} \bar{\sigma}_{t}\right) f_{t, L}-\Phi\left(\Phi^{-1}\left(\bar{p}_{L}\right)-b_{L} \bar{\sigma}_{t}\right)\left[Q_{X_{t}}\left(\bar{p}_{L}\right)\right]^{a_{L}-b_{L}} g_{t, L}, \\
k_{t, G}=\left(h_{t, G}+\left[Q_{X_{t}}\left(\bar{p}_{G}\right)\right]^{a_{G}-b_{G}} g_{t, G}\right)^{-1}, \quad k_{t, L}=\left(h_{t, L}+\left[Q_{X_{t}}\left(\bar{p}_{L}\right)\right]^{a_{L}-b_{L}} g_{t, L}\right)^{-1} .
\end{gathered}
$$

\section{B Convexity in the Loss Domain}

This appendix derives the optimal consumption choice in the case the individual's two-part power utility function is convex in the loss domain; that is, $0 \leq \gamma_{L} \leq 1$. We start with the following proposition.

Proposition 4. Consider an individual with the utility function for gains (12), the utility function for losses (13), and reference level specification (15) who solves the quantile maximization problem (30). Denote by y the Lagrange multiplier associated with the static budget constraint in (30), and by $\widehat{M}_{t}$ the dual state price density. Assume that $0 \leq \gamma_{L} \leq 1$. 
Furthermore, assume that $Q_{\widehat{M}_{t}}\left(1-Z_{\widehat{M}_{t}}\right) / w_{t, G}^{\prime}\left(1-Z_{\widehat{M}_{t}}\right)$ is non-increasing in $Z_{\widehat{M}_{t}}$ and that the function $l_{t}(x)$ as defined in (53) below is convex for every $t \in[0, T]$. Then optimal dual consumption $\widehat{c}_{t}^{*}$ is given by

$$
\left.Q_{\widehat{c}_{t}}^{*}\left(Z_{\widehat{M}_{t}}\right)=Q_{\widehat{c}_{t, G}}^{*}\left(Z_{\widehat{M}_{t}}\right) \mathbb{1}_{\left[Z_{\widehat{M}_{t}} \geq p_{t}^{*}\right.}\right]^{-L_{t} \mathbb{1}}\left[Z_{\widehat{M}_{t}}<p_{t}^{*}\right]
$$

Here,

$$
Q_{\widehat{c}_{t, G}}^{*}\left(Z_{\widehat{M}_{t}}\right)=\left[\frac{e^{\delta t} y Q_{\widehat{M}_{t}}\left(1-Z_{\widehat{M}_{t}}\right)}{\gamma_{G} \widehat{M}_{0} w_{t, G}^{\prime}\left(1-Z_{\widehat{M}_{t}}\right)}\right]^{\frac{1}{\gamma_{G}-1}}
$$

The Lagrange multiplier $y$ and the thresholds $\left\{p_{t}^{*}\right\}_{t \in[0, T]}$ are determined such that 30] is maximized.

Proof. We first note that the assumption that $Q_{\widehat{M}_{t}}\left(1-Z_{\widehat{M}_{t}}\right) / w_{t, G}^{\prime}\left(1-Z_{\widehat{M}_{t}}\right)$ is non-increasing in $Z_{\widehat{M}_{t}}$ is similar to Assumption 4.1 in Jin and Zhou (2008).

If the individual experiences a gain, then optimal dual consumption in the gain domain $Q_{\widehat{c}_{t, G}}^{*}\left(Z_{\widehat{M}_{t}}\right)$ is given by 32 . Because $Q_{\widehat{M}_{t}}\left(1-Z_{\widehat{M}_{t}}\right) / w_{t, G}^{\prime}\left(1-Z_{\widehat{M}_{t}}\right)$ is assumed to be nonincreasing in $Z_{\widehat{M}_{t}}$, it follows that $Q_{\widehat{c}_{t, G}}^{*}(\cdot)$ is a quantile function.

Define now

$$
l_{t}(x)=w_{t, L}\left(n_{t}^{-1}(x)\right)
$$

with $n_{t}(x)=n_{t, 2}\left(n_{t, 1}^{-1}(x)\right), n_{t, 1}(x)=\mathbb{P}\left\{M_{t}>x\right\}$ and $n_{t, 2}(x)=\mathbb{E}\left[M_{t} \mathbb{1}_{\left[M_{t}>x\right]}\right]$.

Zhang, Jin, and Zhou (2011) show that if $l_{t}(x)$ is convex for every $t \in[0, T]$, then optimal dual consumption in the loss domain $Q_{\widehat{c}_{t, L}}^{*}\left(Z_{\widehat{M}_{t}}\right)$ is given by $-L_{t}$. Hence, optimal dual consumption $\widehat{c}_{t}^{*}$ is given by

$$
\widehat{c}_{t}^{*}=Q_{\widehat{c}_{t}}^{*}\left(Z_{\widehat{M}_{t}}\right)=Q_{\widehat{c}_{t, G}}^{*}\left(Z_{\widehat{M}_{t}}\right) \mathbb{1}_{\left[Z_{\widehat{M}_{t}} \geq p_{t}^{*}\right.}-L_{t} \mathbb{1}_{\left[Z_{\widehat{M}_{t}}<p_{t}^{*}\right.},
$$

with $Q_{\widehat{c}_{t, G}}^{*}\left(Z_{\widehat{M}_{t}}\right)$ given by $(32)$. The Lagrange multiplier $y$ and the thresholds $\left\{p_{t}^{*}\right\}_{t \in[0, T]}$ are determined such that 30 is maximized. 
A standard verification (see Karatzas and Shreve (1998), p. 103) shows that the optimal solution obtained from the Lagrangians equals the optimal solution of the quantile problem.

We note that the class of probability weighting functions satisfying condition (53) is not empty; see Zhang et al. (2011, pp. 269-270) who provide sufficient conditions for (53) to hold. In particular, they provide a rich class of probability weighting functions $w_{t, L}(\cdot)$, including a subclass of inverse S-shaped probability weighting functions, for which condition (53) holds.

We summarize the optimal consumption choice in the following theorem.

Theorem 2. Consider an individual with the utility function for gains (12), the utility function for losses (13), reference level specification (15), the probability weighting function for gains satisfying (41) and the probability weighting function for losses satisfying (42) who solves the optimization problem (18). Denote by $\theta_{t}^{*}$ the individual's optimal reference level at time $t$ implied by substituting the optimal past consumption choices into (15), by y the Lagrange multiplier associated with the static budget constraint in 18), and by $\widehat{M}_{t}$ the dual state price density. Assume that $0 \leq \gamma_{L} \leq 1$ and that the function $l_{t}(x)$ as defined in (53) above is convex for every $t \in[0, T]$. Then the optimal consumption choice at time $t$ is given by

$$
c_{t}^{*}=\theta_{t}^{*}+\widehat{c}_{t}^{*},
$$

with

$$
\widehat{c}_{t}^{*}=Q_{\widehat{c}_{t, G}}^{*}\left(1-F_{\widehat{M}_{t}}\left(\widehat{M}_{t}\right)\right) \mathbb{1}_{\left[\widehat{M}_{t} \leq \xi_{t}\right]}-L_{t} \mathbb{1}_{\left[\widehat{M}_{t}>\xi_{t}\right]} .
$$

Here, $\xi_{t} \equiv Q_{\widehat{M}_{t}}\left(1-p_{t}^{*}\right)$ and

$$
Q_{\widehat{c}_{t, G}}^{*}\left(1-F_{\widehat{M}_{t}}\left(\widehat{M}_{t}\right)\right)= \begin{cases}d_{t, 1}\left(\widehat{M}_{t}\right)^{\frac{1-a_{G}}{\gamma_{G}-1}}, & \text { if } \widehat{M}_{t} \leq Q_{\widehat{M}_{t}}\left(\bar{p}_{G}\right), \\ d_{t, 2}\left(\widehat{M}_{t}\right)^{\frac{1-b_{G}}{\gamma_{G}-1}}, & \text { if } \widehat{M}_{t}>Q_{\widehat{M}_{t}}\left(\bar{p}_{G}\right),\end{cases}
$$


where

$$
d_{t, 1}=\left(\frac{y e^{\delta t}}{k_{t, G} \gamma_{G} \widehat{M}_{0}}\right)^{\frac{1}{\gamma_{G}-1}}, \quad d_{t, 2}=\left(\frac{y e^{\delta t}}{k_{t, G}\left[Q_{\widehat{M}_{t}}\left(\bar{p}_{G}\right)\right]^{a_{G}-b_{G}} \gamma_{G} \widehat{M}_{0}}\right)^{\frac{1}{\gamma_{G}-1}} .
$$

The Lagrange multiplier $y$ and the thresholds $\left\{p_{t}^{*}\right\}_{t \in[0, T]}$ are determined such that (30) is maximized.

Proof. By substituting the expression for $w_{t, G}^{\prime}(\cdot)$ (see 41) into 32 and using $Z_{\widehat{M}_{t}} \equiv 1-$ $F_{\widehat{M}_{t}}\left(\widehat{M}_{t}\right)$ and $Q_{\widehat{M}_{t}}\left(F_{\widehat{M}_{t}}\left(\widehat{M}_{t}\right)\right)=\widehat{M}_{t}$, we obtain (57) which is clearly decreasing in $\widehat{M}_{t}$. By Propositions 2 and $3 . \widehat{c}_{t}^{*}=Q_{\widehat{c}_{t, G}}^{*}\left(1-F_{\widehat{M}_{t}}\left(\widehat{M}_{t}\right)\right) \mathbb{1}_{\left[\widehat{M}_{t} \leq Q_{\widehat{M}_{t}}\left(1-p_{t}^{*}\right)\right]}-L_{t} \mathbb{1}_{\left[\widehat{M}_{t}>Q_{\widehat{M}_{t}}\left(1-p_{t}^{*}\right)\right.}$. The optimal consumption choice $c_{t}^{*}$ finally follows from the equivalence relationships between the dual problem and the primal (original) problem summarized in Proposition 1. 


\section{References}

Abdellaoui, M., 2000. Parameter-free elicitation of utility and probability weighting functions. Management Science 46, 1497-1512.

Abdellaoui, M., Bleichrodt, H., L'Haridon, O., 2008. A tractable method to measure utility and loss aversion under prospect theory. Journal of Risk and Uncertainty 36, 245-266.

Abel, A. B., 1990. Asset prices under habit formation and catching up with the joneses. American Economic Review 80, 38-42.

Ang, A., Bekaert, G., Lui, J., 2005. Why stocks may disappoint. Journal of Financial Economics 76, 471-508.

Basak, S., Shapiro, A., 2001. Value-at-risk based risk management: Optimal policies and asset prices. Review of Financial Studies 14, 371-405.

Bell, D. E., 1982. Regret in decision making under uncertainty. Operations Research 30, 961-981.

Bell, D. E., 1983. Risk premiums for decision regret. Management Science 29, 1156-1166.

Bell, D. E., 1985. Disappointment in decision making under uncertainty. Operations Research 33, 1-27.

Benartzi, S., Thaler, R., 1995. Myopic loss aversion and the equity premium puzzle. Quarterly Journal of Economics 110, 73-92.

Benzoni, L., Collin-Dufresne, P., Goldstein, R. S., 2007. Portfolio choice over the life-cycle when the stock and labor markets are cointegrated. Journal of Finance 62, 2123-2167.

Berkelaar, A. B., Kouwenberg, R., Post, T., 2004. Optimal portfolio choice under loss aversion. Review of Economics and Statistics 86, 973-987.

Bilsen, S. van., Bovenberg, A. L., Laeven, R. J. A., 2019a. Consumption and portfolio choice under internal multiplicative habit formation, forthcoming in the Journal of Financial and Quantitative Analysis.

Bilsen, S. van., Laeven, R. J. A., Nijman, Th. E., 2019b. Consumption and portfolio choice under loss aversion and endogenous updating of the reference level, forthcoming in Management Science.

Bleichrodt, H., Pinto, J. L., 2000. A parameter-free elicitation of the probability weighting function in medical decision analysis. Management Science 46, 1485-1496.

Bodie, Z., Merton, R. C., Samuelson, W. F., 1992. Labor supply flexibility and portfolio choice in a life cycle model. Journal of Economic Dynamics and Control 16, 427-449.

Chacko, G., Viceira, L. M., 2005. Dynamic consumption and portfolio choice with stochastic volatility in incomplete markets. Review of Financial Studies 18, 1369-1402.

Cocco, J. F., 2005. Portfolio choice in the presence of housing. Review of Financial Studies 18, 535-567.

Constantinides, G. M., 1990. Habit formation: A resolution of the equity premium puzzle. Journal of Political Economy 98, 519-543.

Cox, J. C., Huang, C., 1989. Optimal consumption and portfolio policies when asset prices follow a diffusion process. Journal of Economic Theory 49, 33-83.

Cox, J. C., Huang, C., 1991. A variational problem arising in financial economics. Journal of Mathematical Economics 20, 465-487.

Detemple, J. B., Karatzas, I., 2003. Non-addictive habits: Optimal consumption-portfolio policies. Journal of Economic Theory 113, 265-285.

Detemple, J. B., Zapatero, F., 1992. Optimal consumption-portfolio policies with habit formation. Mathematical Finance 2, 251-274.

Edwards, R. D., 2008. Health risk and portfolio choice. Journal of Business and Economics Statistics 26, 472-485.

Gomes, F. J., 2005. Portfolio choice and trading volume with loss averse investors. Journal of Business 78 , 675-706.

Gomes, F. J., Kotlikoff, L. J., Viceira, L. M., 2008. Optimal life-cycle investing with flexible labor supply: A welfare analysis of life-cycle funds. American Economic Review 98, 297-303.

Goovaerts, M. J., Laeven, R. J. A., 2008. Actuarial risk measures for financial derivative pricing. Insurance: Mathematics and Economics 42, 540-547. 
Guillén, M., Jørgensen, P. L., Nielsen, J. P., 2006. Return smoothing mechanisms in life and pension insurance: Path-dependent contingent claims. Insurance: Mathematics and Economics 38, 229-252.

Guillén, M., Nielsen, J. P., Pérez-Marín, A. M., Petersen, K. S., 2013. Performance measurement of pension strategies: a case study of danish life-cycle products. Scandinavian Actuarial Journal 2013, 49-68.

Gul, F., 1991. A theory of disappointment aversion. Econometrica 59, 667-686.

He, X. D., Strub, M. S., Zariphopoulou, T., 2019. Forward rank-dependent performance criteria: Time-consistent investment under probability distortion, Working Paper.

He, X. D., Zhou, X. Y., 2011. Portfolio choice via quantiles. Mathematical Finance 21, 203-231.

He, X. D., Zhou, X. Y., 2016. Hope, fear and aspirations. Mathematical Finance 26, 3-50.

Jin, H., Zhou, X. Y., 2008. Behavioral portfolio selection in continuous time. Mathematical Finance 18, 385-426.

Jørgensen, P. L., Linnemann, P., 2011. A comparison of three different pension savings products with special emphasis on the payout phase. Annals of Actuarial Science 6, 137-152.

Kahneman, D., Tversky, A., 1979. Prospect theory: An analysis of decision under risk. Econometrica 47, 263-292.

Karatzas, I., Lehoczky, J. P., Shreve, S. E., 1987. Optimal consumption and portfolio decisions for a "small investor" on a finite horizon. SIAM Journal of Control and Optimization 25, 1557-1586.

Karatzas, I., Shreve, S. E., 1998. Methods of Mathematical Finance, vol. 39. Springer.

Köszegi, B., Rabin, M., 2009. Reference-dependent consumption plans. American Economic Review 99, 909-936.

Labuschagne, C. C. A., Offwood, T. M., 2010. A note on the connection between the Esscher-Girsanov transform and the Wang transform. Insurance: Mathematics and Economics 47, 385-390.

Laeven, R. J. A., Stadje, M. A., 2014. Robust portfolio choice and indifference valuation. Mathematics of Operations Research 39, 1109-1141.

Linnemann, P., Bruhn, K., Steffensen, M., 2014. A comparison of modern investment-linked pension savings products. Annals of Actuarial Science 9, 72-84.

Liu, J., 2007. Portfolio selection in stochastic environments. Review of Financial Studies 20, 1-39.

Loomes, G., Sugden, R., 1982. Regret theory: An alternative theory of rational choice under uncertainty. Economic Journal 92, 805-824.

Loomes, G., Sugden, R., 1986. Disappointment and dynamic consistency in choice under uncertainty. Review of Economic Studies 53, 271-282.

Maurer, R., Mitchell, O. S., Rogalla, R., Siegelin, I., 2016. Accounting and actuarial smoothing of retirement payouts in participating life annuities. Insurance: Mathematics and Economics 71, 268-283.

Merton, R. C., 1969. Lifetime portfolio selection under uncertainty: The continuous-time case. Review of Economics and Statistics 51, 247-257.

Merton, R. C., 1971. Optimum consumption and portfolio rules in a continuous-time model. Journal of Economic Theory 3, 373-413.

Mossin, J., 1968. Optimal multiperiod portfolio policies. Journal of Business 41, 215-229.

Muermann, A., Mitchell, O. S., Volkman, J. M., 2006. Regret, portfolio choice, and guarantees in defined contribution schemes. Insurance: Mathematics and Economics 39, 219-229.

Pagel, M., 2017. Expectations-based reference-dependent life-cycle consumption. Review of Economic Studies 84, $885-934$.

Pliska, S. R., 1986. A stochastic calculus model of continuous trading: Optimal portfolios. Mathematics of Operations Research 11, 371-382.

Quiggin, J., 1994. Regret theory with general choice sets. Journal of Risk and Uncertainty 8, 153-165.

Samuelson, P. A., 1969. Lifetime portfolio selection by dynamic stochastic programming. Review of Economics and Statistics 51, 239-246. 
Samwick, A. A., 1998. Discount rate heterogeneity and social security reform. Journal of Development Economics $57,117-146$.

Schroder, M., Skiadas, C., 2002. An isomorphism between asset pricing models with and without linear habit formation. Review of Financial Studies 15, 1189-1221.

Shao, A. W., Chen, H., Sherris, M., 2019. To borrow or insure? Long term care costs and the impact of housing. Insurance: Mathematics and Economics 85, 15-34.

Sugden, R., 1993. An axiomatic foundation for regret theory. Journal of Economic Theory 60, 159-180.

Sundaresan, S. M., 1989. Intertemporally dependent preferences and the volatility of consumption and wealth. Review of Financial Studies 2, 73-89.

Tversky, A., Kahneman, D., 1992. Advances in prospect theory: Cumulative representation of uncertainty. Journal of Risk and Uncertainty 5, 297-323.

Wang, S. S., 2000. A class of distortion operators for pricing financial and insurance risks. Journal of Risk and Insurance $67,15-36$.

Wu, G., Gonzalez, R., 1996. Curvature of the probability weighting function. Management Science 42, 1676-1690.

Xia, J. M., Zhou, X. Y., 2016. Arrow-debrew equilibria for rank-dependent utilities. Mathematical Finance 26, $558-588$.

Zhang, S., Jin, H. Q., Zhou, X. Y., 2011. Behavioral portfolio selection with loss control. Acta Mathematica Sinica 27, 255-274. 


\section{Figures \& Tables}

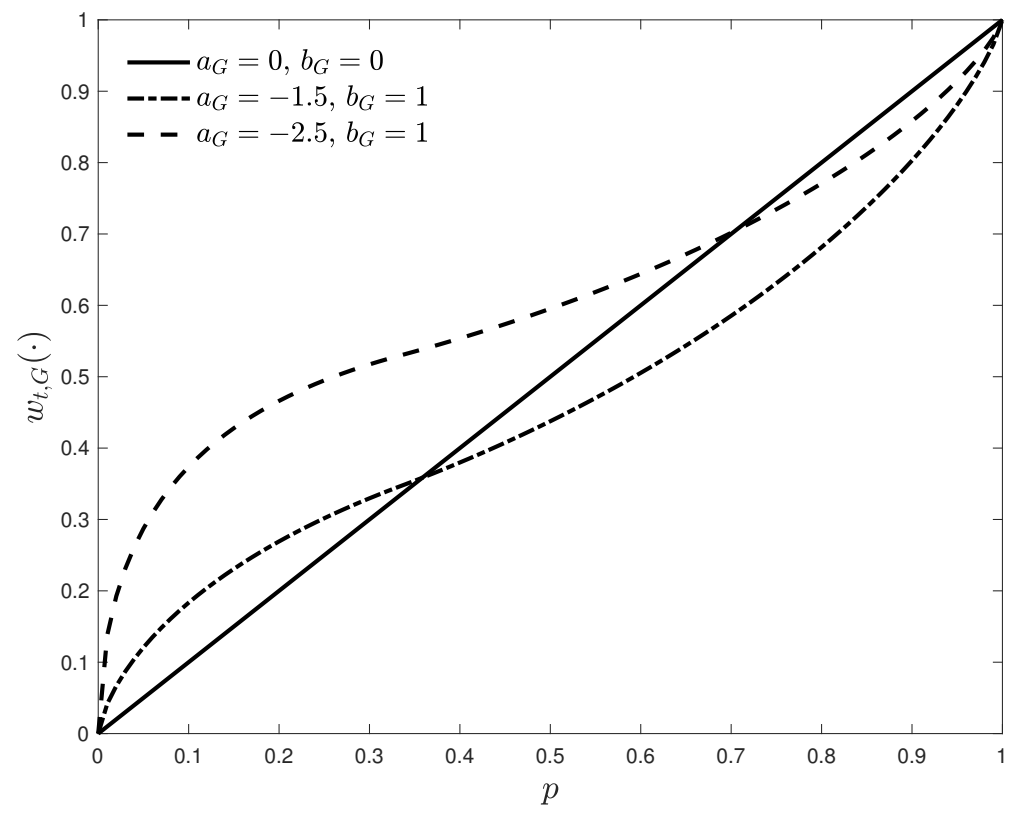

Figure 1: Illustration of probability weighting function. The figure illustrates the probability weighting function $w_{t, G}(\cdot)$ for various sets of parameter values. We assume $\left(\bar{\mu}_{t}, \bar{\sigma}_{t}, \bar{p}_{G}\right)=(-0.30,0.63,0.33)$. 


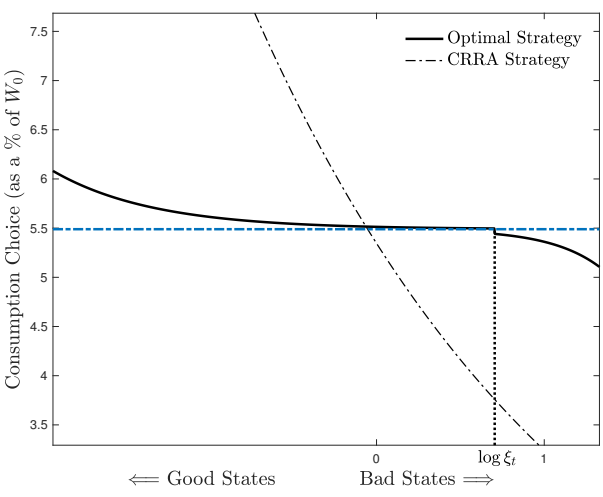

(a) No probability weighting

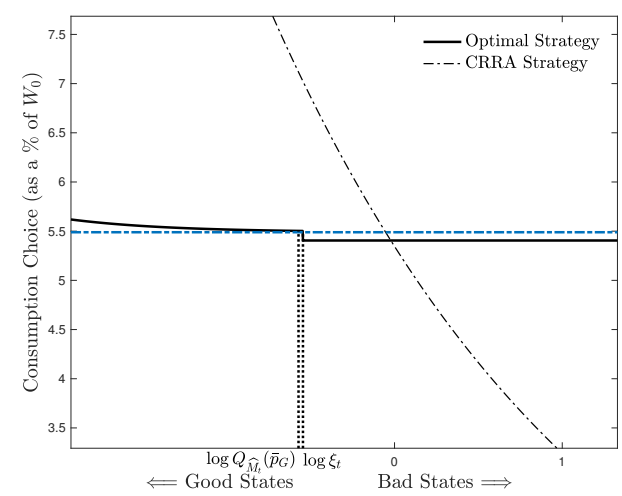

(c) Strong overweighting of unlikely unfavorable events in loss domain $\left(a_{G}=b_{G}=0, b_{L}=1\right)$

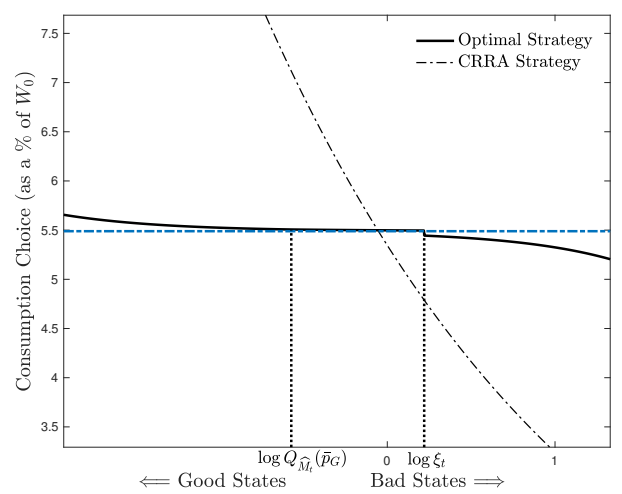

(b) Overweighting of unlikely unfavorable events (b) $\left(a_{G}=0, b_{G}=0.3\right.$ and $\left.b_{L}=0.5\right)$

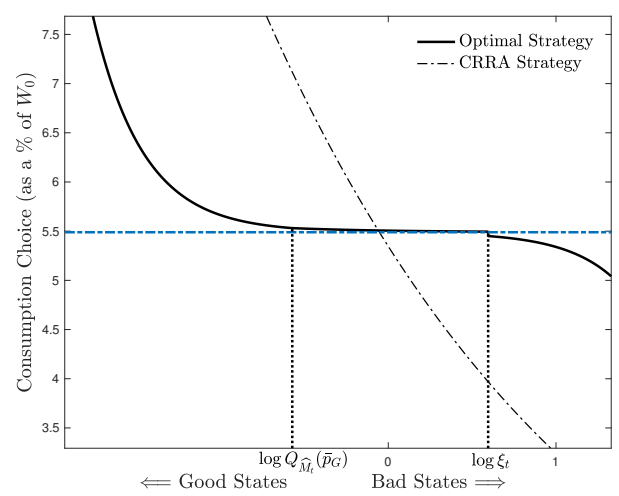

(d) Overweighting of unlikely favorable events

(d) in gain domain $\left(a_{G}=-1, b_{G}=b_{L}=0\right)$

Figure 2: Optimal consumption choice. The figure illustrates the optimal consumption choice (expressed as a percentage of the individual's initial wealth $W_{0}$ ) at time $t=10$ as a function of the log dual pricing kernel for various sets of parameter values. The dashed lines represent the individual's reference level $\theta_{t}$ (expressed as a percentage of the individual's initial wealth $W_{0}$ ). The dash-dotted lines show the optimal consumption choice (expressed as a percentage of the individual's initial wealth $W_{0}$ ) of an individual with CRRA utility (with relative risk aversion equal to 2). We assume $\left(\lambda, r, \kappa, \gamma_{G}, \gamma_{L}, \alpha, \beta, \bar{p}_{G}, \bar{p}_{L}\right)=$ $(0.2,0.01,2,0.4,1.3,0,0,0.33,0.33)$. The maximum possible loss $L_{t}$ is set equal to the individual's reference level $\theta_{t}$. Note that the curvature parameter $a_{L}$ does not play a role. 


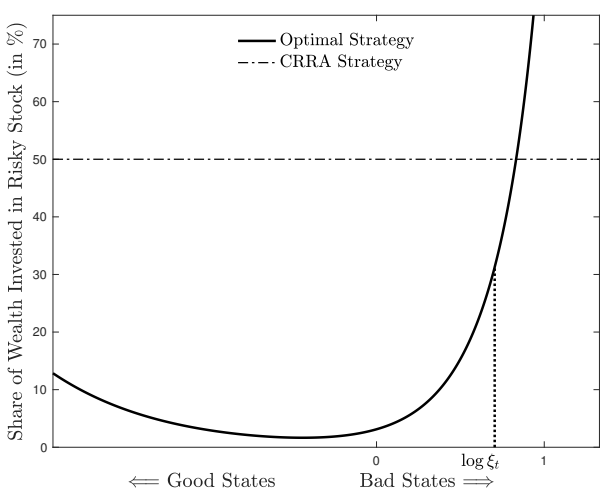

(a) No probability weighting

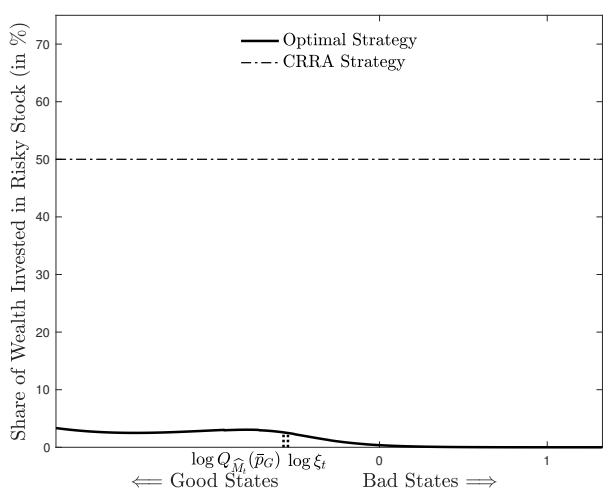

(c) Strong overweighting of unlikely unfavorable events in loss domain $\left(a_{G}=b_{G}=0, b_{L}=1\right)$

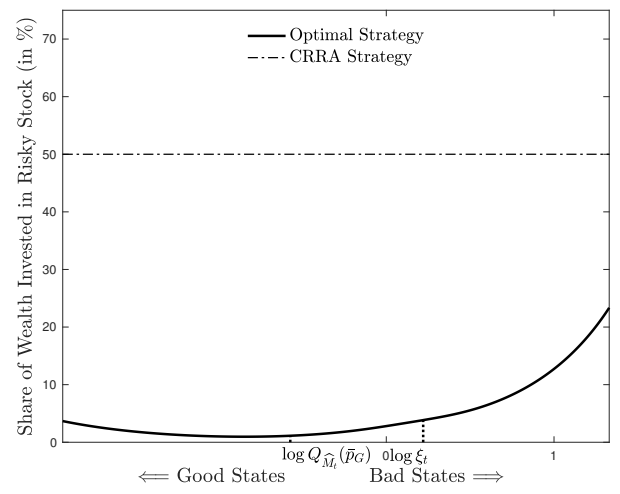

(b) Overweighting of unlikely unfavorable events b) $\left(a_{G}=0, b_{G}=0.3\right.$ and $\left.b_{L}=0.5\right)$

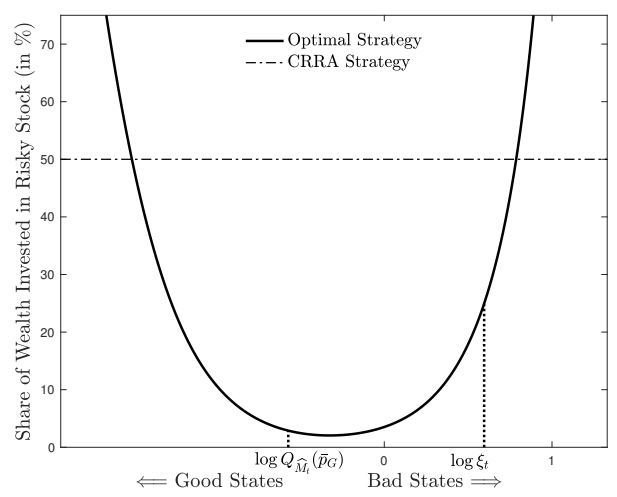

(d) Overweighting of unlikely favorable events

(d) in gain domain $\left(a_{G}=-1, b_{G}=b_{L}=0\right)$

Figure 3: Optimal portfolio choice. The figure illustrates the optimal share of wealth invested in the risky stock at time $t=10$ as a function of the log dual pricing kernel for various sets of parameter values. The dash-dotted lines show the optimal portfolio choice of an individual with CRRA utility (with relative risk aversion equal to 2). We assume $\left(\lambda, r, \kappa, \gamma_{G}, \gamma_{L}, \alpha, \beta, \bar{p}_{G}, \bar{p}_{L}\right)=(0.2,0.01,2,0.4,1.3,0,0,0.33,0.33)$. The maximum possible loss $L_{t}$ is set equal to the individual's reference level $\theta_{t}$. Note that the curvature parameter $a_{L}$ does not play a role. 


\begin{tabular}{cccccc}
\hline & \multicolumn{5}{c}{ Curvature Parameters $\left(\gamma_{G}, \gamma_{L}\right)$} \\
\cline { 2 - 6 } Loss Aversion Index $\kappa$ & $(0.4,1.1)$ & $(0.2,1.3)$ & $(0.4,1.3)$ & $(0.6,1.3)$ & $(0.4,1.5)$ \\
\hline 1 & $1.44(13.7)$ & $0.39(36.7)$ & $0.53(19.6)$ & $1.03(9.9)$ & $0.20(24.7)$ \\
2 & $0.77(34.3)$ & $0.21(64.4)$ & $\mathbf{0 . 2 8}(40.9)$ & $0.50(26.0)$ & $0.11(45.6)$ \\
3 & $0.52(58.1)$ & $0.14(88.2)$ & $0.18(62.5)$ & $0.31(45.8)$ & $0.07(65.1)$ \\
\hline
\end{tabular}

Table 1: Minimum welfare losses due to loss aversion. This table reports the minimum welfare loss (in \%) due to loss aversion. We measure minimum welfare losses in terms of the relative decline in certainty equivalent consumption. The number between brackets denotes the value of the relative risk aversion coefficient that minimizes the difference between the individual's actual utility and the individual's optimal utility. We assume $\left(\lambda, r, \alpha, \beta, a_{G}, a_{L}, b_{G}, b_{L}\right)=(0.2,0.01,0,0,0,0,0,0)$. The maximum possible loss $L_{t}$ is set equal to the individual's reference level $\theta_{t}$.

\begin{tabular}{cc}
\hline Reference Level Parameters $(\alpha, \beta)$ & Welfare Loss (in \%) \\
\hline$(0,0)$ & $0.28(40.9)$ \\
$(0.1,0.1)$ & $0.51(52.0)$ \\
$(0.2,0.2)$ & $1.15(56.6)$ \\
$(0.4,0.4)$ & $4.62(56.0)$ \\
$(0.6,0.6)$ & $13.45(54.3)$ \\
\hline
\end{tabular}

Table 2: Minimum welfare losses due to loss aversion and an endogenous reference level. This table reports the minimum welfare loss (in \%) due to loss aversion and an endogenous reference level. We measure minimum welfare losses in terms of the relative decline in certainty equivalent consumption. The number between brackets denotes the value of the relative risk aversion coefficient that minimizes the difference between the individual's actual utility and the individual's optimal utility. We assume $\left(\lambda, r, \kappa, \gamma_{G}, \gamma_{L}, a_{G}, a_{L}, b_{G}, b_{L}\right)=$ $(0.2,0.01,2,0.4,1.3,0,0,0,0)$. The maximum possible loss $L_{t}$ is set equal to the individual's initial reference level $\theta_{0}$ for every $t$. 


\begin{tabular}{cc}
\hline Curvature Parameters $\left(a_{G}, b_{G}, b_{L}\right)$ & Welfare Loss (in \%) \\
\hline$(0,0,0)$ & $0.28(40.9)$ \\
$(0,0.3,0.5)$ & $0.05(73.0)$ \\
$(0,0,1)$ & $0.02(107.7)$ \\
$(-0.5,0,0)$ & $0.37(38.5)$ \\
$(-1,0,0)$ & $0.73(35.8)$ \\
$(-0.5,0.3,0.5)$ & $0.06(69.2)$ \\
\hline
\end{tabular}

Table 3: Minimum welfare losses due to loss aversion and probability weighting. This table reports the minimum welfare loss (in \%) due to loss aversion and probability weighting. We measure minimum welfare losses in terms of the relative decline in certainty equivalent consumption. The number between brackets denotes the value of the relative risk aversion coefficient that minimizes the difference between the individual's actual utility and the individual's optimal utility. We assume $\left(\lambda, r, \kappa, \gamma_{G}, \gamma_{L}, \alpha, \beta, \bar{p}_{G}, \bar{p}_{L}\right)=$ $(0.2,0.01,2,0.4,1.3,0,0,0.33,0.33)$. The maximum possible loss $L_{t}$ is set equal to the individual's reference level $\theta_{t}$. Note that the curvature parameter $a_{L}$ does not play a role.

\begin{tabular}{cc}
\hline Curvature Parameters $\left(a_{G}, b_{G}, b_{L}\right)$ & Welfare Loss (in \%) \\
\hline$(0,0,0)$ & $4.62(56.0)$ \\
$(0,0.3,0.5)$ & $1.81(75.3)$ \\
$(0,0,1)$ & $1.97(89.5)$ \\
$(-0.5,0,0)$ & $6.87(52.5)$ \\
$(-1,0,0)$ & $15.21(48.2)$ \\
$(-0.5,0.3,0.5)$ & $2.70(70.6)$ \\
\hline
\end{tabular}

Table 4: Minimum welfare losses due to loss aversion, an endogenous reference level and probability weighting. This table reports the minimum welfare loss (in \%) due to loss aversion, an endogenous reference level and probability weighting. We measure minimum welfare losses in terms of the relative decline in certainty equivalent consumption. The number between brackets denotes the value of the relative risk aversion coefficient that minimizes the difference between the individual's actual utility and the individual's optimal utility. We assume $\left(\lambda, r, \kappa, \gamma_{G}, \gamma_{L}, \alpha, \beta, \bar{p}_{G}, \bar{p}_{L}\right)=(0.2,0.01,2,0.4,1.3,0.4,0.4,0.33,0.33)$. The maximum possible loss $L_{t}$ is set equal to the individual's initial reference level $\theta_{0}$ for every $t$. Note that the curvature parameter $a_{L}$ does not play a role. 